\title{
A grid of MARCS model atmospheres for late-type stars
}

\section{Methods and general properties}

\author{
B. Gustafsson ${ }^{1}$, B. Edvardsson ${ }^{1}$, K. Eriksson ${ }^{1}$, U. G. Jørgensen ${ }^{2}$, Å. Nordlund ${ }^{2}$, and B. Plez ${ }^{3,1}$ \\ 1 Department of Physics and Astronomy, Uppsala Astronomical Observatory, Box 515, 751 20 Uppsala, Sweden \\ e-mail: [Bengt.Gustafsson; bg] @astro.uu.se \\ 2 Niels Bohr Institute for Astronomy, Physics and Geophysics, Copenhagen University, Blegdamsveg 17, Copenhagen Ø, 2100 , \\ Denmark \\ 3 GRAAL, Université de Montpellier II, 34095 Montpellier Cedex 05, France
}

Received 5 March 2008 / Accepted 30 April 2008

\section{ABSTRACT}

\begin{abstract}
Context. In analyses of stellar spectra and colours, and for the analysis of integrated light from galaxies, a homogeneous grid of model atmospheres of late-type stars and corresponding flux spectra is needed.

Aims. We construct an extensive grid of spherically-symmetric models (supplemented with plane-parallel ones for the highest surface gravities), built on up-to-date atomic and molecular data, and make it available for public use.

Methods. The most recent version of the MARCS program is used.

Results. We present a grid of about $10^{4}$ model atmospheres for stars with $2500 \mathrm{~K} \leq T_{\text {eff }} \leq 8000 \mathrm{~K},-1 \leq \log g=\log \left(G M / R^{2}\right) \leq$ 5 (cgs) with various masses and radii, $-5 \leq[\mathrm{Me} / \mathrm{H}] \leq+1$, with $[\alpha / \mathrm{Fe}]=0.0$ and 0.4 and different choices of $\mathrm{C}$ and $\mathrm{N}$ abundances. This includes " $\mathrm{CN}$-cycled" models with $\mathrm{C} / \mathrm{N}=4.07$ (solar), 1.5 and $0.5, \mathrm{C} / \mathrm{O}$ ranging from 0.09 to (normally) 5.0 to also represent stars of spectral types $\mathrm{R}, \mathrm{S}$ and $\mathrm{N}$, and with $1.0 \leq \xi_{\mathrm{t}} \leq 5 \mathrm{~km} \mathrm{~s}^{-1}$. We also list thermodynamic quantities $\left(T, P_{\mathrm{g}}, P_{\mathrm{e}}, \rho\right.$, partial pressures of molecules, etc.) and provide them on the World Wide Web, as well as calculated fluxes in approximately 108000 wavelength points. Underlying assumptions in addition to 1D stratification (spherical or plane-parallel) include hydrostatic equilibrium, mixing-length convection and local thermodynamic equilibrium. We discuss a number of general properties of the models, in particular in relation to the effects of changing abundances, of blanketing, and of sphericity. We illustrate positive and negative feedbacks between sphericity and molecular blanketing. We compare the models with those of other available grids and find excellent agreement with planeparallel models of Castelli \& Kurucz (if convection is treated consistently) within the overlapping parameter range. Although there are considerable departures from the spherically-symmetric NextGen models, the agreement with more recent PHOENIX models is gratifying.

Conclusions. The models of the grid show considerable regularities, but some interesting departures from general patterns occur for the coolest models due to the molecular opacities. We have tested a number of approximate "rules of thumb" concerning effects of blanketing and sphericity and often found them to be astonishingly accurate. Some interesting new phenomena have been discovered and explored, such as the intricate coupling between blanketing and sphericity, and the strong effects of carbon enhancement on metal-poor models. We give further details of line absorption data for molecules, as well as details of models and comparisons with observations in subsequent papers.
\end{abstract}

Key words. stars: atmospheres - Sun: abundances - stars: fundamental parameters - stars: general - stars: late-type stars: supergiants

\section{Introduction}

Since the first grids of line-blanketed, model atmospheres for late-type stars were published (Carbon \& Gingerich 1969; Querci et al. 1974; Peytremann 1974; Gustafsson et al. 1975; Kurucz 1979; Johnson et al. 1980), there has been a very impressive improvement in underlying data, in particular, for atomic and molecular absorption. Accurate continuous absorption coefficients for a number of heavy elements have been calculated within the Opacity Project (Seaton et al. 1994), and the Iron Project (Bautista 1997). The Opacity Project also contributed accurate transition probabilities for a wealth of spectral lines for elements where the LS coupling approximation was applicable. Thanks to systematic efforts by Kurucz (see http://kurucz . harvard. edu) line lists with transition probabilities for millions of lines of heavy elements have been calculated. Also, the admirable and systematic work by experimental physicists (e.g.
Blackwell et al. 1989; Nave et al. 1994; Hartman et al. 2003; Lawler et al. 2007) has led to the identification and measurements of many more metal transitions, e.g., from Fe I and Fe II, than existed before. The results of these efforts have been made easily accessible in data bases, such as the Vienna Atomic Line Database, VALD (see Piskunov et al. 1995; Stempels et al. 2001; see also http://www. astro.uu.se/ vald). Additional significant progress has been made in the calculation of accurate damping constants for strong atomic lines (Anstee \& O'Mara 1995; Barklem et al. 2000a) as well as for hydrogen self broadening (Barklem et al. 2000b).

Impressive progress has also been made in the study of molecular absorption starting from practically nothing 30 years ago (see Jørgensen 1994b, 2005, for reviews). Very extensive line lists have thus been calculated for most diatomic and polyatomic molecules that contribute opacity in stellar atmospheres. The lists are based on laboratory measurements of wavelengths 
and $g f$ values with simple theoretical extensions, or are the result of more extensive quantum-mechanical ab-initio calculations. Even if the absorption cross-sections are not always of satisfactory quality as yet, the existence of these lists makes it possible to calculate models for, e.g., $\mathrm{M}$ and $\mathrm{C}$ stars that are realistic enough to compare reasonably well with observations.

Simultaneous with these improvements of basic physics, steps to improve the physical consistency of the models have also been taken. The traditional assumptions of plane-parallel stratification in homogenous layers, of stationary hydrostatic equilibrium, mixing-length convection and local thermodynamic equilibrium (LTE) have stepwise been possible to relax. Grids of spherically-symmetric models for giants and supergiants were made by Plez et al. (1992) and Jørgensen et al. (1992). Dynamic pulsating model atmospheres for cool giants have been developed by Wood (1979) and Bowen (1988), and these were improved with time-dependent dust formation by Fleischer et al. (1992) and Höfner \& Dorfi (1997), and further developed to include frequency-dependent radiative transfer by Höfner (1999) and Höfner et al. (2003). Nordlund \& Stein and collaborators (Nordlund 1982; Nordlund \& Dravins 1990; Stein \& Nordlund 1998; Asplund et al. 1999, 2000a) developed 3D simulations with proper hydrodynamics and radiation fields taken into account for solar-type stars, and Freytag (2001) developed full "star-in-a-box" models for supergiants. These simulations show a striking agreement with observations of solar granulation and spectral line profiles for solar type stars, and clearly demonstrate the qualitative difference between traditional 1D models and reality: while the temperature structure in the upper layers of the $1 \mathrm{D}$ models is determined by radiative cooling and heating, the radiative heating in real late-type stars is balanced to a significant extent also by expansion cooling of upwelling gas.

A consistent treatment of radiative transfer in models for late-type stars without making the assumption of LTE is complicated due to the great number of atomic and molecular species affecting the radiative field; the wealth of levels and transitions in these species; and the lack of basic data for these transitions not the least of which is the lack of cross sections for atomic and molecular collisions with electrons and hydrogen atoms. From the first attempt to construct a reasonably realistic nonLTE model for a late-type star (the Sun) made by Anderson (1989), the development of algorithms and computers has now made it possible to calculate grids of such models (Hauschildt et al. 2002). The lack of accurate collision cross sections, however, is still a major problem in these efforts.

In the present paper we present an extensive grid of model atmospheres for late-type stars. The models are still classical, in the sense that they are one-dimensional, i.e., spherically symmetric or plane-parallel, with LTE assumed and convection taken into account by using the standard mixing-length theory. In spite of the inadequacy of these underlying physical assumptions, which must be abandoned for detailed analysis of late-type stellar spectra at high accuracy, we still think the present grid will be useful for years to come for various applications extending from studies of individual stars to galactic evolution and populations in external galaxies.

The grid extends from models for A-type stars to M, S, and C star models, from dwarfs to supergiants in luminosity, from $10^{-5}$ times solar to 10 times solar in metallicity, and with various choices of parameters like radius and mass, abundances of $\mathrm{C}, \mathrm{N}$, and $\mathrm{O}$, of “ $\alpha$ elements" ( $\mathrm{Ne}, \mathrm{Mg}, \mathrm{Si}, \mathrm{S}, \mathrm{Ar}, \mathrm{Ca}, \mathrm{Ti}$ ) relative to $\mathrm{Fe}$, and of microturbulence. We also provide model spectral energy distributions (SEDs) sampled in about $10^{5}$ frequency points. With respect to methods and underlying data used, the model grid is homogeneous.

The grid is presented in a number of papers. In this initial paper, we (Sect. 2) sketch the development of our computer code MARCS, from which models produced at different earlier stages are in wide use, give a general outline of the methods now used (Sects. 3 and 4), and underlying data (Sect. 5), as well as properties of the grid (Sect. 6). Models are also compared with models from other contemporary grids (Sect. 7). In subsequent papers, we shall discuss models for A-G stars (Edvardsson et al., Paper II), K and R stars (Eriksson et al., Paper III), M stars (Plez et al., Paper IV), S stars (Plez et al., Paper V), C stars (Jørgensen et al., Paper VI), and models for very metal-poor stars (Paper VII). In these subsequent papers, the most important new opacity data are described; the model structures are described and analysed; and the model SEDs, fluxes and colours are compared to some observational data to explore and illustrate the applicability of the models. The total number of models is about $10^{4}$. The details of these models, including their calculated fluxes at different wavelengths, are available via http: // marcs.astro.uu.se.

\section{The development of MARCS}

Since the early 1970ies, we have developed and used the code MARCS for constructing late-type model atmospheres. Spectral-line blanketing was first considered using opacity distribution functions (ODFs, Gustafsson et al. 1975) and this technique and its underlying ODF approximation was proven to be fully adequate for F, G, and $\mathrm{K}$ stars. Thus, models could be calculated with just a few hundred frequency points and extensive grids for $\mathrm{G}$ and $\mathrm{K}$ giants (Bell et al. 1976) as well as R stars (Olander 1981) were issued on the basis of the ODF approximation. The ODFs were constructed with a program called SSG, originally devised for synthetic colour calculations by Bell (see, e.g., Bell 1971). The line list used by this program was based on laboratory wavelengths and oscillator strengths, as well as "astrophysical oscillator strengths" derived from the solar spectrum. The total list contained about 50000 lines. The SSG program was used next to calculate model spectra and colours, which were systematically compared with observations (e.g. Gustafsson \& Bell 1979; Bell \& Gustafsson 1989). From these comparisons, a generally good agreement was found, which gave us the confidence to apply the models in a number of studies, e.g., of abundances in globular cluster stars. However, there was a mismatch in certain wavelength regions, in particular in the ultraviolet and violet, where the models were obviously too bright. We tentatively ascribed this discrepancy to the absence in the line lists and the models of many weak lines, which together affect the stellar spectra.

There were also more fundamental problems with the ODF method. One was that the ODFs had to be recalculated when the chemical composition was changed, which made it inflexible for stars with special and peculiar abundances, such as carbon stars. To circumvent this, a special method to add ODFs for individual molecules was invented (Saxner \& Gustafsson 1984). However, for N-type carbon star models, we also found that the effects of molecules in the upper atmospheric layers, contributing strong opacity at wavelengths different from the atomic absorption at greater depths, made the ODF approximation unsatisfactory (cf., Ekberg et al. 1986). To provide satisfactory analysis for a study of the chemical composition of carbon stars (Lambert et al. 1986), we consequently changed the scheme to opacity sampling, in which the adequate absorption 
at each monochromatic wavelength point is treated in full detail (yet, assuming LTE in our case), following Peytremann (1974) and Sneden et al. (1976). In order to obtain an accurate representation of the total radiation contribution to the heat balance and the radiative pressure force, this required an increase of the number of frequency points by one to two orders of magnitude. A number of model grids were calculated with this new program, for carbon stars (cf., Lambert et al. 1986; Jørgensen et al. 1992), for M giants (Plez et al. 1992) and M dwarfs (Brett \& Plez 1993) and applied to studies of individual stars. We gradually extended the underlying line list by calculating and adopting more complete data for diatomic molecules, such as $\mathrm{TiO}$ (Jørgensen 1994a; Plez et al. 1992; Plez 1998), as well as polyatomic molecules like HCN (Jørgensen et al. 1985; Jørgensen 1990), $\mathrm{C}_{2} \mathrm{H}_{2}$ (Jørgensen thesis; cited in Jørgensen 1989) and $\mathrm{H}_{2} \mathrm{O}$ (Plez et al. 1992; Alvarez \& Plez 1998; Jørgensen et al. 2001; Decin et al. 2000).

In order to provide models for a major effort to study the build-up of chemical elements in the Galactic disk using solartype stars, we further extended the line list, adding the great number of metal lines from Kurucz's calculations (Plez et al. 1992; Edvardsson et al. 1993). This version of the code was also used in calculating a grid for hydrogen-poor carbon stars $(\mathrm{R} \mathrm{Cr}$ B stars, Asplund et al. 1997), and applied in an abundance study of such stars (Asplund et al. 2000b). Somewhat different versions of the code were used to compare models to ISO spectra and other IR spectra, partly for calibration purposes of the satellite instrumentation, of F, G, and $\mathrm{K}$ stars (van der Bliek et al. 1996, and subsequent papers; Decin et al. 2003, and references therein) as well as of M stars (Alvarez et al. 2000; Fluks et al. 1994) and carbon stars (Loidl et al. 2001). We also made comparisons of model colours with observed ones (Bessell et al. 1998), and applied colours and SEDs for establishing temperature scales (see, e.g., Massey et al. 2007, and references therein).

The development of MARCS has always been driven by our own (and collaborators') needs for models for studies of particular stars, mainly for abundance determination. It has been a flexible "laboratory set-up", rather than a "common-user instrument", and we have been reluctant to calculate models for stars of types, which we cannot explore ourselves to understand the limitations of our models. Until now, less than $10^{3}$ models have been published, which is a small number compared to the number actually calculated and used. Also, several different versions of MARCS have appeared, which has led to some confusion because of inhomogeneities among the grids produced. It was, therefore, judged important to develop a common updated version, and to use it to construct and publish an extensive homogeneous grid. The results of this effort is presented in this and subsequent papers.

\section{Physical assumptions and equations}

The basic assumptions have been listed above. We shall not discuss their adequacy further here (for a review, see, e.g., Gustafsson \& Jørgensen 1994), but comment on them one at a time, and in connection with that list some of the corresponding fundamental equations, for further reference below.

\subsection{Stratification and hydrostatic equilibrium}

Assuming spherical symmetry we may write the equation of hydrostatic equilibrium:

$\nabla P_{\text {tot }}=-\rho \frac{G M_{\mathrm{r}}}{r^{2}}$
$P_{\text {tot }}$ being the total pressure, $\rho$ the matter density, $G$ Newton's constant of gravity, and $M_{\mathrm{r}}$ the stellar mass inside radius $r$. We neglect the atmospheric mass in comparison with the total stellar mass $M$, and thus assume $M_{\mathrm{r}}=M$. For $\nabla P_{\text {tot }}$ we have

$\nabla P_{\text {tot }}=\nabla P_{\mathrm{g}}+\nabla P_{\text {turb }}+\nabla P_{\text {rad }}$,

where $P_{\mathrm{g}}$ and $P_{\text {turb }}$ are the gas pressure and turbulent pressure, respectively, and the last term is the force exerted by the radiation,

$\nabla P_{\mathrm{rad}}=-\frac{1}{c} \int_{0}^{\infty}\left(\kappa_{\lambda}+\sigma_{\lambda}\right) F_{\lambda} \mathrm{d} \lambda$

where $F_{\lambda}$ is the radiative energy flux per wavelength unit, $\kappa_{\lambda}$ and $\sigma_{\lambda}$ are the monochromatic absorption and scattering coefficients, respectively, and $c$ is the speed of light. The boundary condition for Eq. (2) is

$P_{\mathrm{g}}(r=\infty)=P_{\text {turb }}(r=\infty)=0.0$.

The formulation of the boundary condition involving the radiative force is, however, not quite trivial in practice; see Plez et al. (1992). For the turbulent pressure $P_{\text {turb }}$ one may write

$P_{\text {turb }}=\beta \rho v_{\mathrm{t}}^{2}$

where $\rho$ is the gas density and $v_{\mathrm{t}}$ a characteristic velocity. This pressure is measuring the force produced by the kinetic movements of the gas, whether due to convective or other turbulent gas motions. The parameter $\beta$ is $\sim 1$, with an exact value depending on whether the motions occur more or less isotropically. With our general lack of knowledge about $v_{\mathrm{t}}$ it is reasonable to adopt an approximate recipe for $P_{\text {turb }}$. We begin with assuming a depth independent value of $v_{\mathrm{t}}$. The dominant depth variation in $P_{\mathrm{g}}=\mathcal{R} \rho T / \mu_{\mathrm{mol}}, \mathcal{R}$ being the gas constant, occurs in $\rho$. Neglecting the variation in the temperature $T$ and in the mean molecular weight $\mu_{\mathrm{mol}}$ as well as in $v_{\mathrm{t}}$ and in $M_{\mathrm{r}}=M$, one finds

$\nabla P_{\mathrm{g}} \approx-\rho \frac{G M}{r^{2}}\left(\frac{1-\frac{1}{4 \pi c} \frac{\chi_{\mathrm{F}}}{G}(L / M)}{1+\beta \frac{\mu_{\mathrm{mol}}}{\mathcal{R} T} v_{\mathrm{t}}^{2}}\right)$,

where $\chi_{\mathrm{F}}$ is the flux-weighted mean of the extinction coefficient per gram and $L$ the luminosity of the star. Here, we have also assumed that the dominating fraction of the stellar flux is carried by radiation, i.e. the approximation is most accurate for the upper radiative zones of the models. One may regard the right-hand side of Eq. (6) as the local effective surface gravity $g_{\text {eff }}$ times $\rho$. We thus find that we can mimic the effects of the radiative force and/or the turbulent pressure on the models by using models with those effects neglected with an adjusted gravity:

$g_{\mathrm{eff}}=g\left(\frac{1-\frac{1}{4 \pi c} \frac{\chi_{\mathrm{F}}}{G}(L / M)}{1+\beta \frac{\mu_{\mathrm{mol}}}{\mathcal{R} T} v_{\mathrm{t}}^{2}}\right)$,

where

$g=g(r)=\frac{G M}{r^{2}}$

Defining the effective Eddington luminosity, $L_{\text {Edd }}^{\text {eff }} \equiv 4 \pi G M c / \chi_{\mathrm{F}}$, we obtain

$g_{\mathrm{eff}}=g\left(\frac{1-\left(L / L_{\mathrm{Edd}}^{\mathrm{eff}}\right)}{1+\beta \gamma\left(v_{\mathrm{t}} / c_{\mathrm{s}}\right)^{2}}\right)$,

where $c_{\mathrm{s}}=\sqrt{\gamma P_{\mathrm{g}} / \rho}$ is the sound spead for an ideal gas and $\gamma$ is the adiabatic index. Basically, we have neglected the depth variation in $\gamma\left(v_{\mathrm{t}} / c_{\mathrm{s}}\right)^{2}$ in deriving this expression. 
Thus, a model with a turbulent velocity $v_{\mathrm{t}}$ may be represented by a model with a reduced gravity $g_{\text {eff }}$ and $v_{\mathrm{t}}=0$, according to this recipe. Similarly, effects from the radiation force may be mimicked by changes in $g$ or $M$. However, the Planck mean may vary strongly with depth and with stellar fundamental parameters, and one should therefore be careful in the use of Eq. (7) for exploring the radiative effects on the atmospheric structure and, e.g., the possible effects on mass-loss rates. For a more detailed study for red supergiants and asymptotic-branch stars, see Gustafsson \& Plez (1992) and Jørgensen \& Johnson (1992), respectively. We have tested the use of Eq. (7) to simulate the effects of turbulent pressure for a number of models at various points in the grid and find that it leads to very small errors in the temperature structure (less than $5 \mathrm{~K}$ in the temperature throughout the model for a depth independent $v_{\mathrm{t}}$ in the interval 0 to $10 \mathrm{~km} \mathrm{~s}^{-1}$ ). We, therefore, have chosen to set $v_{\mathrm{t}}=0$ for all grid models, and advise those who would have liked a different choice to use models with a different mass or $g$, according to the recipe given in Eq. (7). It should be noted that the mixinglength treatment of convection adopted here (see Sect. 2.2 below) leads to a rapidly varying formal convective velocity, in particular close to the boundary where Schwarzschild stability sets in. If this variation were included the term $\nabla P_{\text {turb }}=\nabla\left(\beta \rho v_{\mathrm{t}}^{2}\right)$ would get a major contribution from $\nabla v_{\mathrm{t}}$. However, more realistic simulations of convection show that $v_{\mathrm{t}}$ varies much less with depth than $\rho$, in accordance with what was assumed in the derivation of Eq. (7) above.

The acceleration of gravity, $g$, for our spherical models is a depth-varying quantity, according to Eq. (8). Also the stellar energy flux $F(r)$ and $T_{\text {eff }}$ are varying with depth. Thus, we have

$F(r)=F_{\text {rad }}(r)+F_{\text {conv }}(r)=\sigma_{\mathrm{SB}} T_{\text {eff }}(r)^{4}$,

where $F_{\text {rad }}$ and $F_{\text {conv }}$ are the radiative and convective flux, respectively, and $\sigma_{\mathrm{SB}}$ is Stefan-Boltzmann's constant. What remains constant (in stationary models) is the mass $M$ (since only a tiny fraction of the total stellar mass resides in the atmosphere), and the luminosity, $L=4 \pi r^{2} F(r)$. We label the models by the values of $T_{\text {eff }}$ and $g$ at a radius $r=R_{1}$ where $\tau_{\text {Ross }}$, the opticaldepth scale based on the Rosseland mean opacity, is equal to 1.0, i.e.:

$T_{\text {eff }} \equiv T_{\text {eff }}\left(R_{1}\right)=\left(\frac{L}{4 \pi R_{1}^{2}}\right)^{\frac{1}{4}}$,

$g \equiv \frac{M G}{R_{1}^{2}}$

\subsection{Mixing length convection}

We have used the version of the mixing-length "theory" as presented by Henyey et al. (1965). The convective energy flux is given by

$F_{\text {conv }}=\frac{1}{2} \rho C_{\mathrm{p}} T v_{\text {conv }} \frac{\ell}{H_{\mathrm{p}}} \delta \Delta$.

Here,

$\delta \Delta=\frac{\Gamma}{(1+\Gamma)}\left(\nabla_{\mathrm{T}}-\nabla_{\mathrm{ad}}\right)$,

where

$$
\begin{aligned}
\Gamma & =v_{\text {conv }} \rho C_{\mathrm{p}} \frac{1+y\left(\rho \chi_{\text {Ross }} \ell\right)^{2}}{8 \sigma_{\mathrm{SB}} T^{3} \rho \chi_{\mathrm{Ross}} \ell}, \\
\nabla_{\mathrm{T}} & =\frac{\mathrm{d} \ln T}{\mathrm{~d} \ln P},
\end{aligned}
$$

and $H_{\mathrm{p}}$ is the local pressure scale height,

$H_{\mathrm{p}}=\frac{P}{g \rho}$.

In the above, $\nabla_{\mathrm{ad}}$ is the adiabatic temperature gradient; $C_{\mathrm{p}}$ the specific heat at constant pressure; $\chi_{\text {Ross }}$ the Rosseland mean opacity; and $\ell$ the mixing length. Equation (13) is valid if $\left(\nabla_{\mathrm{T}}-\right.$ $\left.\nabla_{\mathrm{ad}}\right) \geq 0$. Also,

$v_{\text {conv }}=\frac{\ell}{H_{\mathrm{p}}} \sqrt{\frac{G M}{r^{2}} H_{\mathrm{p}} Q \delta \Delta / v}$

with

$Q=-\frac{T}{\rho}\left(\frac{\partial \rho}{\partial T}\right)_{\mathrm{p}}$,

the derivative taken at constant thermodynamic pressure. In addition to the mixing length parameter, $\alpha=\ell / H_{\mathrm{p}}$, there are two more explicit parameters in this formulation: $y$, which is related to the adopted temperature distribution within the convective elements, and $v$, which deals with the energy dissipation by the turbulent viscosity. As a standard, we have chosen the parameters according to the suggestions by Henyey et al.: $\alpha=1.5$, $y=0.076$ and $v=8$. It should be noted that different choices than these are made, sometimes without being pointed out, in other current work. The effects of varying the convective parameters were explored for models of red giants by Gustafsson et al. (1975). However, these variations do not at all map the real possible range of errors due to our (inadequate) treatment of the convective energy transport and do not give relevant information on the effects of the thermal inhomogeneities generated by convection. The convective flux is added to the radiation energy flux, and we may then write the energy equilibrium equation

$F_{\text {conv }}+F_{\text {rad }}=\frac{L}{4 \pi r^{2}}$

\subsection{LTE and radiative transfer}

All number densities of all atoms and molecules are assumed to follow from the corresponding laws for thermal equilibrium; the Saha equation; and the corresponding equation of chemical equilibrium for molecules (Gibson \& Heitler 1928; see also Russell 1934). Similarly, all excitation equilibria and all partition functions are calculated adopting the Boltzmann distribution, with higher terms in the atomic partition functions cut according to the method of Irwin (1981).

The radiation source function is assumed to be

$S_{\lambda}=\frac{\kappa_{\lambda}}{\kappa_{\lambda}+\sigma_{\lambda}} B_{\lambda}(T)+\frac{\sigma_{\lambda}}{\kappa_{\lambda}+\sigma_{\lambda}} J_{\lambda}$,

where $B_{\lambda}(T)$ is the Planck function. All line absorption is assumed to occur in true absorption, i.e.,

$\kappa_{\lambda}=\kappa_{\lambda}^{\text {cont }}+\kappa_{\lambda}^{\text {line }}$

where $\kappa_{\lambda}^{\text {cont }}$ and $\kappa_{\lambda}^{\text {line }}$ are the sums of all continuous absorption contributions and line absorption contributions, respectively. The mean intensity $J_{\lambda}$ is calculated from

$J_{\lambda}=\int_{0}^{1} j_{\lambda}(\mu) \mathrm{d} \mu$.

Here, following Feautrier (1964), we define $j_{\lambda}(\mu)$ as

$j_{\lambda}(\mu)=\frac{1}{2}\left(I_{\lambda}(\mu)+I_{\lambda}(-\mu)\right)$ 
where $I_{\lambda}(\mu)$ is the specific intensity in the direction specified by $\mu=\cos \theta, \theta$ being the angle relative to a stellar radius. The equation of radiative transfer for $j_{\lambda}$ is

$\frac{\mathrm{d}^{2} j_{\lambda}}{\mathrm{d} \tau_{\lambda}^{2}}=j_{\lambda}-S_{\lambda}$

where $\tau_{\lambda}$ is measured along the ray. For a discussion of adequate boundary conditions for $j_{\lambda}$ and their implementation, see Nordlund (1984). Knowing $j_{\lambda}(\mu)$, the wavelength-integrated flux in the radial direction can be calculated from a derivative of the second Eddington moment $K_{\lambda}$, defined by

$K_{\lambda}=\int_{0}^{1} \mu^{2} j_{\lambda} \mathrm{d} \mu$.

Thus, we have for the monochromatic flux

$F_{\lambda}=4 \pi\left(\frac{\partial K_{\lambda}}{\partial \tau_{\lambda}}-\frac{1}{r} \frac{3 K_{\lambda}-J_{\lambda}}{\kappa_{\lambda}+\sigma_{\lambda}}\right)$,

cf., Mihalas (1978, his Eq. (2.80)) and then

$F_{\text {rad }}=\int F_{\lambda} \mathrm{d} \lambda$,

which is used in the energy conservation equation, Eq. (20). Alternatively, the energy balance may be expressed as

$\frac{\mathrm{d}}{\mathrm{d} r}\left(\left(F_{\text {rad }}+F_{\text {conv }}\right) \cdot r^{2}\right)=0$.

For $F_{\text {conv }}=0$, which is often the case in the upper layers of the models, this is equivalent to

$\int \kappa_{\lambda}\left[J_{\lambda}\left(\tau_{\text {Ross }}\right)-B_{\lambda}\left(T\left(\tau_{\text {Ross }}\right)\right)\right] \mathrm{d} \lambda=q_{\text {rad }}-q_{\text {thermal }}=0$,

with

$q_{\mathrm{rad}} \equiv \int \kappa_{\lambda} J_{\lambda}\left(\tau_{\mathrm{Ross}}\right) \mathrm{d} \lambda$

$q_{\text {thermal }} \equiv \int \kappa_{\lambda} B_{\lambda}\left(T\left(\tau_{\text {Ross }}\right)\right) \mathrm{d} \lambda$.

\section{Physical data}

The volume of physical data needed in the calculation of model atmospheres of late-type stars is considerable. Data are needed for the calculation of the ionisation equilibrium of atoms and the dissociation equilibrium of molecules. These data include chemical composition data, ionisation energies and dissociation energies, as well as partition functions. Moreover, continuous absorption and scattering coefficients are needed. The, by far, most extensive and most demanding need, however, is the various data that are necessary for the proper calculation of the line absorption. Here, we shall briefly present the data used to calculate ionisation-dissociation equilibria as well as continuous absorption and scattering. Some more details concerning the lineabsorption data are given in Papers II-VII.

The basic chemical composition adopted is that of the Sun, as listed by Grevesse et al. (2007). There is still some dispute on the $\mathrm{C}, \mathrm{N}$, and $\mathrm{O}$ abundances adopted there $(\mathrm{C}=8.39$, $\mathrm{N}=7.78$, and $\mathrm{O}=8.66$ ) and, therefore, we have taken the data of Grevesse \& Sauval (1998) with CNO abundances higher by about 0.2 dex for an alternative solar-metallicity sub-grid. The effects of these differences are explored in Sect. 6.3. In varying the overall metallicity of the models $([\mathrm{Me} / \mathrm{H}])$, we have as a standard scaled the abundances of all elements heavier than $\mathrm{He}$ in unison. The abundances of so-called $\alpha$ elements $\mathrm{O}, \mathrm{Ne}, \mathrm{Mg}, \mathrm{Si}$, $\mathrm{S}, \mathrm{Ar}, \mathrm{Ca}$, and Ti tend to vary somewhat differently for galactic stars (see, e.g., Edvardsson et al. 1993; Reddy et al. 2003; Ryde \& Lambert 2004; Nissen et al. 2004; Cayrel et al. 2004). The variation, however, is not necessarily the same for all stellar populations in the Galaxy (see, e.g., Fuhrmann 1998; and Bensby et al. 2005) or in other galaxies (see Venn et al. 2004). Therefore, we offer models with two different sets of abundances: a set with a uniform scaling for each element with $[\mathrm{Me} / \mathrm{H}]$; and a set where the $\alpha$ elements have been scaled as follows: $[\alpha / \mathrm{Fe}]=0.4$ for $-5.0 \leq[\mathrm{Me} / \mathrm{H}] \leq-1.0 ;[\alpha / \mathrm{Fe}]=-0.4 \times[\mathrm{Me} / \mathrm{H}]$ for $-1.0 \leq$ $[\mathrm{Me} / \mathrm{H}] \leq 0.0$; and $[\alpha / \mathrm{Fe}]=0.0$ for $[\mathrm{Me} / \mathrm{H}] \geq 0.0$. For the giant stars, the results of the first dredge up of CNO processed material is known to lead to a reduced carbon abundance and a correspondingly increased $\mathrm{N}$ abundance (cf. e.g., Boothroyd \& Sackmann 1999). We therefore also offer models with revised C and $\mathrm{N}$ such that $\mathrm{C} / \mathrm{N}=1.5$ and 0.5 by number, respectively, as alternatives to the solar value of $\mathrm{C} / \mathrm{N}=4.07$, though keeping $\mathrm{C}+\mathrm{N}$ constant (cf. Sect. 6.3). For the corresponding giant stars, the changed $\mathrm{C} / \mathrm{N}$ ratio is usually accompanied by a decrease in the isotopic ratio ${ }^{12} \mathrm{C} /{ }^{13} \mathrm{C}$. For the " $\mathrm{CN}$ processed" models we have therefore changed this ratio from solar to 20 and 4, respectively. Finally, we also present models with increased $\mathrm{C}$ abundances for the $\mathrm{M}$ stars and carbon stars such that $\mathrm{C} / \mathrm{O}$ ranges from 0.54 to 0.99 ( $\mathrm{M}$ and $\mathrm{S}$ star models) to above 1.0 (models for C stars).

We use the atomic partition functions and ionisation energies from Irwin (1981) with some modifications. The molecular equilibria were calculated using the partition functions and dissociation energies of Sauval (private communication), which is an update of Sauval \& Tatum (1984) for diatomic molecules, and Irwin (1988, and private communication) for polyatomic molecules. Altogether 519 molecules were included in the equilibrium calculations. A comparison with equilibria calculated by Piskunov (private communication) shows a very good agreement.

The continuous absorption from $\mathrm{HI}, \mathrm{H}^{-}, \mathrm{H}_{2}^{-}, \mathrm{H}_{2}^{+}, \mathrm{He} \mathrm{I}, \mathrm{He}^{-}$, C I, C II, $\mathrm{C}^{-}, \mathrm{N}_{\mathrm{I}}, \mathrm{N}_{\text {II }}, \mathrm{N}^{-}, \mathrm{O}_{\mathrm{I}}, \mathrm{O}_{\mathrm{II}}, \mathrm{O}^{-}, \mathrm{Mg}_{\mathrm{I}}, \mathrm{Mg}_{\mathrm{II}}, \mathrm{Al}$ I, $\mathrm{Al} \mathrm{II}$, $\mathrm{Si}$ I, Si II, Ca I, Ca II, Fe I, and Fe II, as well as $\mathrm{CH}, \mathrm{OH}, \mathrm{CO}^{-}$, and $\mathrm{H}_{2} \mathrm{O}^{-}$were calculated from sources, according to Table 1 .

Corrections were made to the data for CI, MgI, Al I, Si I to account for the fact that TOP base photon cross sections are published with theoretical energy levels derived from the model atom, which depart significantly from the much better known data from laboratory work. For these four species, corrections were considered necessary since they significantly affect the UV fluxes of the models. We identified the individual TOP base energy levels for these species and shifted them to the empirical energies given by the National Institute of Standards and Technology (NIST) data base (http://physics.nist. gov/PhysRefData/ASD/index.html). The TOP base gives the photon absorption cross section (in Mbarn) as a function of the energy of the ejected electron. This electron energy was converted to the energy of the absorbed photon by adding the ionisation energy from the specific electronic level.

The cross-section data for all levels was summed up, assuming LTE for a number of temperatures, and plotted at the full original wavelength resolution at different temperatures. Also collision-induced absorption of $\mathrm{H} \mathrm{I}+\mathrm{H} \mathrm{I}, \mathrm{H} \mathrm{I}+\mathrm{He} \mathrm{I}, \mathrm{H}_{2}+\mathrm{H} \mathrm{I}$, $\mathrm{H}_{2}+\mathrm{H}_{2}$, and $\mathrm{H}_{2}+\mathrm{He}$ I was included. $\mathrm{H}_{2}-\mathrm{He}$ and $\mathrm{H}_{2}-\mathrm{H}_{2}$ CIA data from Borysow et al., referred to in Table 1, are available from http:www.stella.nbi.dk/pub/scan. More extensive 
Table 1. Sources of data for continuous opacities.

\begin{tabular}{|c|c|}
\hline Ion and process & Reference \\
\hline H I b-f ${ }^{1}, f-f^{1}$ & Karzas \& Latter $(1961)^{\ddagger}$ \\
\hline $\mathrm{H}^{-} \mathrm{b}-\mathrm{f}$ & Wishart (1979) \\
\hline$H^{-} f-f$ & Bell \& Berrington (1987) \\
\hline He I b-f & TOPbase $^{2}$ \\
\hline He I f-f & Peach (1970) \\
\hline $\mathrm{He}^{-} \mathrm{f}-\mathrm{f}$ & John (1994) \\
\hline C I, C II b-f & TOPbase $^{\dagger}$ \\
\hline C I, C II f-f & Peach (1970) \\
\hline $\mathrm{C}^{-} \mathrm{f}-\mathrm{f}$ & Bell et al. (1988) \\
\hline N I, N II b-f & TOPbase \\
\hline $\mathrm{N}^{-} \mathrm{f}-\mathrm{f}$ & Ramsbottom et al. (1992) \\
\hline O I, O II b-f & TOPbase \\
\hline $\mathrm{O}^{-} \mathrm{f}-\mathrm{f}$ & John $(1975 a, b)$ \\
\hline $\operatorname{Mg}$ I, Mg II b-f & TOPbase $^{\dagger}$ \\
\hline $\operatorname{Mg}$ I f-f & Peach (1970) \\
\hline Al I, Al II b-f & TOPbase $^{\dagger}$ \\
\hline Si I, Si II b-f & $\mathrm{TOPbase}^{\dagger}$ \\
\hline Si I f-f & Peach (1970) \\
\hline Ca I, Ca II b-f & TOPbase \\
\hline Fe I b-f & TOPbase, Bautista (1997) \\
\hline Fe II b-f & TOPbase \\
\hline other metals $f-f$ & Peach (1970), hydr. approx. \\
\hline $\mathrm{H}_{2}^{+} \mathrm{f}-\mathrm{f}$ & Mihalas (1964) \\
\hline $\mathrm{H}_{2}^{-} \mathrm{f}-\mathrm{f}$ & John (1975a,b), John \& Williams (1975) \\
\hline $\mathrm{CH} b-\mathrm{f}$ & Kurucz et al. (1987) \\
\hline $\mathrm{OH} b-\mathrm{f}$ & Kurucz et al. (1987) \\
\hline $\mathrm{CO}^{-} \mathrm{f}-\mathrm{f}$ & John (1975a,b) \\
\hline $\mathrm{H}_{2} \mathrm{O}^{-} \mathrm{f}-\mathrm{f}$ & John $(1975 a, b)$ \\
\hline $\mathrm{H}$ I+H I CIA ${ }^{3}$ & Doyle (1968) \\
\hline $\mathrm{H}$ I+He I CIA & Gustafsson \& Frommhold (2001) \\
\hline $\mathrm{H}_{2}+\mathrm{H}$ I CIA & Gustafsson \& Frommhold (2003) \\
\hline $\mathrm{H}_{2}+\mathrm{H}_{2} \mathrm{CIA}$ & Borysow et al. (2001) \\
\hline $\mathrm{H}_{2}+\mathrm{He}$ I CIA & Jørgensen et al. (2000) \\
\hline H I scattering & Dalgarno, quoted by Gingerich (1964) \\
\hline $\mathrm{H}_{2}$ scattering & Dalgarno \& Williams (1962) \\
\hline $\mathrm{e}^{-}$scattering & Mihalas (1978) \\
\hline He I scattering & Bues \& Wehrse (1976) \\
\hline
\end{tabular}

1 "b-f" and "f-f" denote bound-free and free-free processes, respectively.

2 "TOPbase": data from the Opacity Project (Seaton et al. 1994), available via http://vizier.u-strasbg.fr/topbase/home. html.

3 "CIA": collision induced or quasi-molecular absorption.

* Modified using the occupation probability formalism, see text.

$\dagger$ The calculated wavelengths of UV and blue C I, Mg I, Al I and Si I absorption edges have been shifted to their. laboratory values, see text.

data sets for the CIA are publicly accessible from http://wwW . astro.ku.dk/ aborysow. Continuous electron scattering as well as Rayleigh scattering from $\mathrm{HI}, \mathrm{H}_{2}$ and $\mathrm{He}$ I was included and assumed to be isotropic.

Line absorption data for atoms and first ions were originally adopted from VALD-1 (Piskunov et al. 1995), but recently data for the ten most important species were modified according to VALD-2 (Stempels et al. 2001). The updated species are Si I, Ca I, Ca II, Ti II, Cr I, Cr II, Mn II, Fe I, Fe II, and Ni II. The VALD-data adopted include the very numerous line data calculated by Kurucz \& Bell (1995, see also http://kurucz. harvard.edu), including lines between predicted energy levels that are not yet experimentally verified. The $g f$ values were, however, modified for 948 lines from VALD-1 and 1276 lines from VALD-2 in the wavelength range 3783 to $8968 \AA$, based on fits of synthetic solar intensity spectra. These model spectra were calculated with the Holweger \& Müller (1974) model and compared with the observed solar disk-centre FTS spectrum of Brault \& Neckel (1987), see Paper II. The model spectrum was required to match the observed equivalent widths to within 0.1 dexin $\log g f$. The resulting $g f$ values are listed at http://marcs . astro.uu. se. Line absorption data for diatomic and polyatomic molecules were considered for species according to Table 2. Most line lists of Jørgensen and collaborators are described by Jørgensen (1997) and may be obtained by anonymous ftp via www. stella.nbi.dk/pub/scan. Line lists for $\mathrm{HCN}, \mathrm{C}_{2} \mathrm{H}_{2}$ and $\mathrm{C}_{3}$ will be added to this data base later in connection with the paper on carbon-enriched stars in the present series. The line lists of Plez referred to in Table 2 as "unpublished" are available via http://marcs . astro.uu.se. Some of these lists were especially tailored for the present grid; in such cases a more detailed description of the line list for the particular species is to be found in one of the subsequent papers in the present series. For the OH A-X system we did not use the data of Gillis et al. (2001) since the Kurucz (1995b) list includes higher vibration and rotation states. For $\mathrm{MgH}$ we did not include the new data of Skory et al. (2003) for ${ }^{24} \mathrm{MgH}$ since we were anxious to be consistent with the $\mathrm{MgH}$ lines for other $\mathrm{Mg}$ isotopes.

Hydrogen line and bound-free opacity, and their merging, were modelled using a code by Barklem (http: //www . astro . uu. se/ barklem/hlinop.html), which is based on the occupation probability formalism of Däppen et al. (1987). The details of the description of this line opacity are given by Barklem \& Piskunov (2003). For the atomic lines of metals, the damping wings were also calculated, using the best available data (Anstee \& O'Mara 1995; Barklem et al. 2000a, and references therein, 2000b; Barklem \& Aspelund-Johansson 2005; and Barklem, private communication). For weak lines where such data were missing we just adopted the Unsöld recipe (cf., Unsöld 1955, his Eq. (76.43)), with a constant enhancement factor set to 2.0 for Na I, 1.3 for Si I, 1.8 for Ca I, 1.4 for Fe I and 2.5 for all other species. (The damping constants given in the line list of Kurucz, which were obtained by perturbation theory, might have been preferred to the use of Unsöld values.) For the molecules, damping was not taken into account - the lack of proper damping parameters makes any such attempt questionable. Also, the huge density of molecular lines often makes the damping wings of a line less important since the intensity of the Doppler cores of neighbouring lines dominate.

In general, it should be noted that although the present atomic and molecular data are very extensive, and much more complete and accurate than, e.g., a decade ago, they are far from perfect. Considerable improvements are still needed, both by adding many more faint lines and by determining line strengths more accurately. The risk that present models are still underblanketed must be appreciated.

\section{Numerical methods}

\subsection{The general method}

Equations (1)-(3), (13), (20) or (30), (27), and (28) with relevant boundary conditions form a closed system of equations for determining the dependent variables $F_{\text {conv }}, T, P_{\mathrm{g}}, P_{\text {rad }}, P_{\text {turb }}, P_{\mathrm{e}}$, as well as quantities like $v_{\text {conv }}, F_{\text {rad }}$, or alternatively $q_{\text {rad }}$. In addition to these quantities, we also need to calculate quantities characteristic (in LTE at least) of the local temperature and pressure: $\kappa(\lambda), \sigma(\lambda), H_{\mathrm{p}}, Q, \nabla_{\mathrm{ad}}$ and $\gamma$. The system of equations is solved 
Table 2. Sources of data for molecular line opacities.

\begin{tabular}{|c|c|}
\hline Species & Reference \\
\hline $\mathrm{HCN}$ vib-rot & $\begin{array}{l}\text { Harris et al. (2002), } \\
\text { Jørgensen et al. (2001) }\end{array}$ \\
\hline $\mathrm{H}_{2} \mathrm{O}$ vib-rot & Barber et al. (2006) \\
\hline $\begin{array}{c}\mathrm{C}_{2} \text { Phillips, Swan, } \\
\text { Ballik-Ramsay }\end{array}$ & Querci et al. (1971, priv. comm.) \\
\hline $\mathrm{C}_{3}$ & Jørgensen et al. (1989) \\
\hline $\mathrm{C}_{2} \mathrm{H}_{2}$ & Jørgensen (1989) \\
\hline $\mathrm{CH}$ vib-rot & Jørgensen et al. (1996) \\
\hline CH A-X, B-X, C-X & Plez et al. (2008) \\
\hline CN A-X, B-X & Plez (unpublished) \\
\hline $\mathrm{CO}$ vib-rot & Goorvitch (1994) \\
\hline CO A-X & Kurucz (1995) \\
\hline CaH A-X, B-X & Plez (unpublished) \\
\hline $\mathrm{FeH} \mathrm{F}^{4} \Delta-\mathrm{X}^{4} \Delta$ & Plez (unpublished) \\
\hline $\mathrm{MgH} \mathrm{A}-\mathrm{X}, \mathrm{B}^{\prime}-\mathrm{X}$ & Kurucz (1995a) \\
\hline NH A-X & Kurucz (1995a) \\
\hline OH vib-rot & Goldman et al. (1998) \\
\hline OH A-X & Kurucz (1995a) \\
\hline $\mathrm{SiH} \mathrm{A}-\mathrm{X}$ & Kurucz (1995a) \\
\hline $\mathrm{SiO}$ vib-rot & Langhoff \& Bauschlicher (1993) \\
\hline $\begin{array}{c}\mathrm{TiO} \alpha, \beta, \gamma, \gamma^{\prime}, \delta, \epsilon, \phi, \\
\mathrm{E}^{3} \Pi-\mathrm{B}^{3} \Pi, \mathrm{a}^{1} \Delta-\mathrm{f}^{1} \Delta\end{array}$ & Plez (1998) \\
\hline VO A-X, B-X, C-X & Plez (unpublished) \\
\hline $\begin{array}{l}\mathrm{ZrO} \mathrm{B}^{1} \Pi-\mathrm{A}^{1} \Delta, \mathrm{B}^{1} \Pi-\mathrm{X}^{1} \Sigma, \\
\quad \mathrm{C}^{1} \Sigma-\mathrm{X}^{1} \Sigma \mathrm{E}^{1} \Phi-\mathrm{A}^{1} \Delta, \\
\mathrm{b}^{3} \Pi-\mathrm{a}^{3} \Delta, \mathrm{d}^{3} \Phi-\mathrm{a}^{3} \Delta, \\
\mathrm{e}^{3} \Pi-\mathrm{a}^{3} \Delta, \mathrm{f}^{3} \Delta-\mathrm{a}^{3} \Delta,\end{array}$ & Plez et al. (2003) \\
\hline
\end{tabular}

on a $\tau_{\text {Ross }}$ scale, with $\kappa_{\text {Ross }}$ calculated by integration over all $\sim 10^{5}$ wavelength points. This choice of depth scale leads to temperature structures that are only moderately affected by changes in the fundamental stellar parameters. Basically, a standard multidimensional Newton-Raphson method is applied to solve the highly non-linear system. All equations are first discretised, in the variables $\tau_{\text {Ross }}$ and $\lambda$. Next, all the resulting equations are linearized in the dependent variables listed above. The input variables at the calculation of thermodynamic quantities and absorption coefficients are $T$ and $P_{\mathrm{e}}$, which is a very appropriate choice as long as $\mathrm{H}^{-}$is a dominating opacity source. (For the coolest models, e.g., $P_{\mathrm{H}}$ or $P_{\mathrm{g}}$ could have been more advantageous.) As a result of the linearisation, a set of linear equations in the variations of the dependent variables is obtained. The coefficients of this system contain derivatives of a great number of quantities relative to the dependent variables. First, a starting model is adopted to make a first calculation of the coefficients possible. Next, the system of linear equations is solved numerically, the resulting variations are applied to the dependent variables, new coefficients are calculated, and a new solution is obtained for the variations. The elimination scheme is basically that of Rybicki (1971), also applied by Gustafsson \& Nissen (1972) and Gustafsson et al. (1975), with an elimination work that scales linearly with the number of wavelength points, $n_{\lambda}$.

\subsection{Radiative transfer}

The total radiative flux, $F_{\text {rad }}, q_{\mathrm{rad}}$ and $\nabla_{\mathrm{r}} P_{\text {rad }}$ are non-local functionals of the model structure and can be calculated from a solution of the transfer equation, Eq. (25). For the model structure obtained after each iteration, we need an accurate solution of the equation with the source function $S_{\lambda}$ given by Eqs. (21) and (23), which then enables us to calculate $F_{\text {rad }}$ and $q_{\text {rad }}$ for that structure. These quantities are used to calculate the corrections (right hand

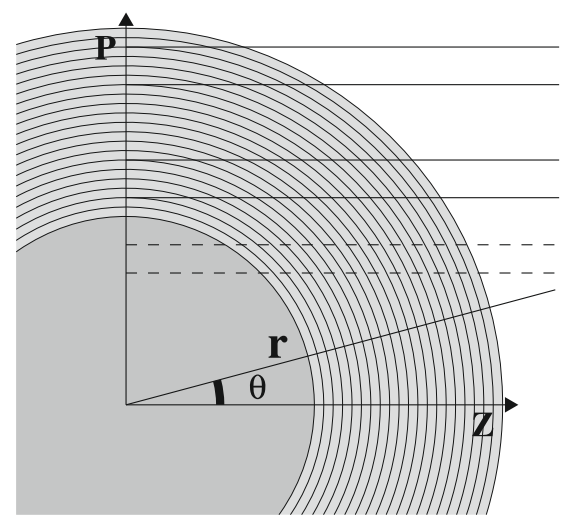

Fig. 1. Sketch of the geometric representation used for solving the spherically-symmetric radiative transfer problem. The full radius mesh used for representing the variables as functions of radius (about 7 points per decade in $\tau_{\mathrm{r}}$ ) is indicated along the radius vector by a set of concentric circles. The distance of rays from the centre of symmetry is measured by the impact parameter $p$. About 15 rays with impact parameters $\leq R_{\mathrm{c}}$, the core radius, are used and are represented here by two dashed lines. The set of rays that do not hit the core, typically about 50 rays but dependent of wavelength, are here represented by five parallel rays. The mesh of points used for solving the equation of radiative transfer are the crossing points between the circles and the horizontal lines. Distances along the rays are measured by $z=\mu r$ where $\mu=\cos \theta$.

sides) in the next Newton-Raphson iteration. We also need a linearisation of $F_{\text {rad }}$ and $q_{\text {rad }}$ in $T$ and $P_{\mathrm{e}}$ to calculate factors, symbolically written as, e.g., $\delta F_{\text {rad }}\left(\tau_{i}\right) / \delta T\left(\tau_{k}\right)$, or $\delta q_{\text {rad }}\left(\tau_{i}\right) / \delta P_{\mathrm{e}}\left(\tau_{k}\right)$, to be used in the coefficient matrix in the forth-coming iteration. Here, $\tau_{i}$ and $\tau_{k}$ are any two radial optical depths in the stellar atmosphere. I.e., we need to estimate how the radiation contribution to the energy balance at each point in the atmosphere is affected by variations in temperature or pressure anywhere else. Both these tasks are accomplished with the iterative method of Nordlund (1984), which was applied to model atmosphere calculations by Plez et al. (1992) for M giants and by Jørgensen et al. (1992) for carbon stars. Here, we shall only give a brief summary for reference.

The equation of radiative transfer is solved along a set of parallel rays, which cover a suitable range of impact parameters, chosen individually for each wavelength (cf. Fig. 1). In practice, about 15 rays equidistant in $\mu$ (the cosine of the angle relative to the normal) are chosen for impact parameters $<R_{\mathrm{c}}$, where $R_{\mathrm{c}}$ is the radius for the stellar non-transparent "core". This core is defined by a radial $\tau_{\lambda} \sqrt{\frac{\kappa_{\lambda}}{\kappa_{\lambda}+\sigma_{\lambda}}}>200$, to allow a great enough thermalization depth for scattered light. Rays that never hit this core, but only pass through the transparent "atmospheric" region, are all chosen to go through pre-selected $\tau_{\text {Ross }}$ points in the vertical scale along a line through the stellar centre and perpendicular to the ray. These rays are distributed with about 5 rays per decade in $\tau_{\lambda}$ along this line.

Scattering is handled by an iterative technique where the radiative transfer equation along a particular single ray is used to correct residual errors. This technique uses a "form factor" $f_{\lambda}$,

$f_{\lambda}(r)=J_{\lambda} / j_{\lambda}$.

Here, both $J_{\lambda}$ and $j_{\lambda}$ can be taken from the previous iteration, since their ratio is not very dependent on the detailed shape of the source function; in this respect, the method is analogous to the variable Eddington technique of Auer \& Mihalas (1970) in handling the angular dependence of the radiative field. After a few iterations a correct solution is obtained. When linearising the 
energy equilibrium equation (Eqs. (20) or (30)) we linearise the transfer equation along a representative ray and apply the form factor, as calculated in the preceding iteration. This relates the changes in the energy balance to changes of the specific intensity along the ray. We adopt this relation as typical for all rays. The final result is then a coupling of the energy equilibrium at each point in the atmosphere to temperatures and electron pressures all over the model.

The representative ray is chosen in the following way: at the radius in the discretisation of the $\tau_{\text {Ross }}$ scale where $\tau_{\lambda}$, measured radially is about $\sqrt{\left(\kappa_{\lambda}+\sigma_{\lambda}\right) / \kappa_{\lambda}}$ the set of rays hits the sphere at a set of angles relative to the normal. We take the ray where the cosine for this angle is close to $1 / \sqrt{3}$, a natural choice in view of the result of the Eddington approximation that the outgoing intensity from a gray atmosphere with a linear source function in this direction has a characteristic depth of formation which can be taken as an average depth of formation for all disk rays.

In order to obtain a sufficient numerical accuracy we linearise the radiative flux directly (Eq. (20)) only for optical depths $\tau_{\text {Ross }}>0.01$, while for smaller depths we linearise the divergence of the flux (i.e. Eq. (30)). This avoids the loss of accuracy associated with the nearly constant monochromatic fluxes at most wavelengths in the optically thin layers. In the discretisation in $\tau_{\text {Ross }}$ we use 56 points, distributed between $\tau_{\text {Ross }}=10^{-5}$ and $\tau_{\text {Ross }}=100$.

The integrals over $\lambda$ are calculated using the trapezian rule. In the $\lambda$ discretisation, we use 10000 points during the first iterations. In the final iterations there are 108000 points, set to produce a sampling density $R \equiv \lambda / \delta \lambda=20000$, with $\lambda_{\min }=910 \AA$ for models warmer than $3500 \mathrm{~K}$ and $1300 \AA$ for cooler models, and $\lambda_{\max }=20 \mu \mathrm{m}$. We have made a series of numerical experiments to investigate the errors resulting from the sampling of the spectrum with a resolution smaller than what corresponds to the characteristic line widths. We did this investigation by making a number of models, each with 30000 wavelength points. The models were identical, except for the particular wavelength points which were sampled differently. We then found the temperature errors for $\tau_{\text {Ross }} \geq 10^{-4}$ to be less than $0.003 \times T_{\text {eff }} \mathrm{K}$ with the maximum errors in the upper layers of the model (above $\tau_{\text {Ross }}=0.01$ ). The corresponding errors in the flux integrals and flux derivative integrals (i.e., the integrals in Eqs. (28) and (30)) by the sampling procedure are less than $1.5 \%$. For the grid models with 108000 wavelength points, the sampling errors are correspondingly smaller (by approximately a factor of $\left.(108000 / 30000)^{-1 / 2}\right)$. They then lead to temperature errors of about $0.002 \times T_{\text {eff }} \mathrm{K}$ or less. For the $\mathrm{M}$ and $\mathrm{C}$ star models, we have found the corresponding errors in fluxes and temperatures to be reduced by a factor of three or more. This is a result of the overlapping molecular-line absorption, which reduces the dominance of a few strong metal lines in the flux derivative integrals in the upper model layers, lines which otherwise demand a high density of sampling points if no special measures are taken to cover them properly by quadrature points.

It should be noted that the fluxes, even if they are monochromatically precise within the model constraints, may be far from representative for the regions surrounding each wavelength point in the spectrum. Thus, model fluxes integrated to represent narrow spectral regions, $\Delta \lambda$ wide, will have relative errors of typically $0.3 \times[\lambda /(R \Delta \lambda)]^{1 / 2}$. E.g., for $100 \AA$ wide bands at $5000 \AA$, the sampling errors will correspond to standard deviations of typically $1.6 \%$. Obviously, synthetic spectra more detailed than the model fluxes must be calculated for theoretical calibrations of, e.g., narrow-band photometry. We have included the surface fluxes in all 108000 wavelength points into the model files published on http: //marcs . astro .uu . se - more detailed highresolution synthetic spectra will also be gradually added.

For the integration of the $\mu$ moments of the radiative intensity (in practice $j_{\lambda}$ ) we use the $\mu$ points defined by the angles in the crossing points between the different parallel rays with impact parameter $p_{i}$ and the concentric spheres around the stellar centre with radii $r_{k}$ corresponding to the discretisation of the vertical $\tau_{\text {Ross }}$ scale, $\tau_{k}$ cf. Fig. 1. I.e., $\mu_{i k}=\left(r_{k}^{2}-p_{i}^{2}\right)^{1 / 2} / r_{k}$, directions for which $j_{\lambda}(\mu)$ is directly available. Considering the character of the radiation field in a spherical atmosphere, this is an adequate set of $\mu$ points. This, despite the fact that they are different for different radii and different wavelengths. The typical number of $\mu$ points is 6 in the plane-parallel case and typically 40-60 in the spherical models. The quadrature in $\mu$ is performed using piece-wise cubic splines, as described by Nordlund (1984).

\subsection{Representation of line opacity}

The line opacities have been pretabulated in the following way: for each individual species (e.g. H I, C I, C II, CH, $\mathrm{C}_{2}$, etc.) and the full set of wavelength points, arrays of line absorption were calculated for a number of $T-P_{6}$ points (or $T$ points for the molecules). The value of $P_{6}$ is given by

$P_{6}=P(\mathrm{HI})+0.42 P(\mathrm{He} \mathrm{I})+0.85 P\left(\mathrm{H}_{2}\right)$

where the static dipole polarisabilities and mean velocities of $\mathrm{He} \mathrm{I}$ and $\mathrm{H}_{2}$ relative to that of $\mathrm{H}$ I have been used to approximately scale the broadening for $\mathrm{H}$ I to these species. Altogether, $17 T$ values and $18 P_{6}$ values were chosen. Tables were constructed for 6 different values of the microturbulence parameter, $\xi_{\mathrm{t}}=0,1,2,5,10$, and $20 \mathrm{~km} \mathrm{~s}^{-1}$. Next, for a given chemical composition and microturbulence parameter, the tables were summed for all atoms and ions over the different species with due consideration to the ionisation equilibria. This gave one table of the summed atomic line absorption at each lambda point for each $T, P_{6}$, and $\xi_{\mathrm{t}}$ chosen. For each molecular species we produced a table for a combination of $12 T$ and $6 \xi_{\mathrm{t}}$ values. In the subsequent model-atmosphere calculations, we found the logarithmic line absorption coefficients from these tables by spline interpolation to the appropriate temperatures and logarithmic pressures. (If models are needed at microturbulence parameters different from those of the tables, interpolation can be made.)

In the calculation of opacity data tables, we added the relevant absorption for each spectral line at each $T$ and $P_{6}$ value to the table for wavelengths points progressing to the blue and the red side of the central line wavelength as far as the line opacity exceeded a predefined cross section. Also, for very weak lines, we then included each line at a minimum of two wavelength points. To limit the total computing time needed and still guarantee that no significant opacity was lost, these limiting cross sections were empirically determined for groups of atoms and ions.

\subsection{Computing time, starting model, and convergence}

The total computing time needed for the calculation of a model atmosphere (the pre-calculation of the absorption coefficient tables excluded) scales approximately as follows: (1) for the radiative transfer part of the problem the time is proportional to $n_{\mu} \times n_{\tau} \times n_{\lambda} ;(2)$ for the calculation of ionisation equilibria and molecular equilibria the time scales as $n_{\tau}$; and (3) for the calculation of absorption coefficients as $n_{\tau} \times n_{\lambda}$. In addition, (4) some 
initiation time is needed. Setting $N_{\tau}=n_{\tau} / 28, N_{\lambda}=n_{\lambda} / 10^{5}$ and $N_{\mu}=n_{\mu} / 6$ and noting that we usually set $n_{\mu}=6$ in the planeparallel case and that $n_{\mu} \approx 2 / 3 \times n_{\tau}$ in the spherical case, we find empirically that the time $t_{\text {it }}$ needed per iteration is, in seconds,

$t_{\text {it }} \approx 10+10 N_{\tau}+5 N_{\lambda}+10 N_{\tau} N_{\lambda}+2 N_{\tau} N_{\lambda} N_{\mu}$.

For a typical spherical model with 108000 wavelength points about $80 \mathrm{~s}$ per iteration are needed on a Mac Pro quad Intel Xeon $2.66 \mathrm{GHz}$ computer for one processor. For a plane-parallel model this is reduced to about $60 \mathrm{~s}$. The relatively small reduction reflects the fact that interpolation in the line-absorption tables (the $N_{\tau} N_{\lambda}$ term) constitutes a major fraction of the computing time; for a highly spherical case (when $N_{\mu}$ becomes great) only, the radiative transfer calculations dominate. No doubt, the calculations of absorption coefficients and their derivatives could be sped up further, e.g., by pre-tabulation. This would primarily be of interest in the calculation of extensive model grids, or for models with more complex physics than described by the approximations in Sect. 3, e.g., with regard to hydrodynamics.

In multi-dimensional Newton-Raphson schemes like the present one, convergence is rapid, provided that a starting solution, which is close enough to the final solution, has been chosen. When model calculations are started from scratch with the MARCS program, usually a gray starting model is chosen for the radiative zone. When the calculated flux in the convectively unstable zone of the first starting model exceeds the total flux, the temperature gradient is automatically and directly reduced, beginning at the onset of the convective instability until the convective flux is smaller than the prescribed total flux. In the present grid, we usually start from a nearby model in the fundamental-parameter space. If the starting model has a different effective temperature, a simple scaling of the temperature structure $T\left(\tau_{\text {Ross }}\right)$ may be applied, but this is not necessary if steps of only a few hundred $\mathrm{K}$ are taken in $T_{\text {eff }}$.

The route towards convergence is often rapid, but not always quadratical. The pronounced non-linearities, not the least in the temperature dependencies of the molecular equilibria with strong effects on the opacities may slow down the convergence if one is not very close to the final solution. Also, the changing presence and depth of the convective zone with temperature and metallicity affect the convergence. Usually, convergence to temperature corrections of less than a few $\mathrm{K}$, and logarithmic pressure corrections smaller than $0.01 \mathrm{dex}$, is obtained all through the models after 4 to 10 iterations. For models with $T_{\text {eff }} \geq 4000 \mathrm{~K}$, the convergence is close to quadratic with corrections reduced by almost one order of magnitude from one iteration to the next. For the cooler models the convergence is slower but still fast. For some parameter choices, however, converged models were not obtained. In particular, this is the case for models with a strong radiative pressure gradient (i.e., close to the effective Eddington limit, see Gustafsson \& Plez 1992) which is close to upsetting the hydrostatic equilibrium condition, i.e., models with high temperatures and low gravities. Another difficulty occurs for models in a small band in the $T_{\text {eff }}-\log g$ diagram, extending from about $T_{\text {eff }}=8000 \mathrm{~K}, \log g=5$, to $T_{\text {eff }}=6750 \mathrm{~K}, \log g=3$, where convergence is not achieved in the deepest layers of the models because the convectively unstable regions are swapping between thin convective zones and zones extending to depths below the bottom of the model. This difficulty may be circumvented by extending the depth scale of the model to deeper layers. Some of the models, though not fully converged in the deepest layers, are nevertheless presented in the grid since the spectrum forming regions (above $\tau_{\text {Ross }}=10$ ) are not affected at all. Convergence problems occurred for some of

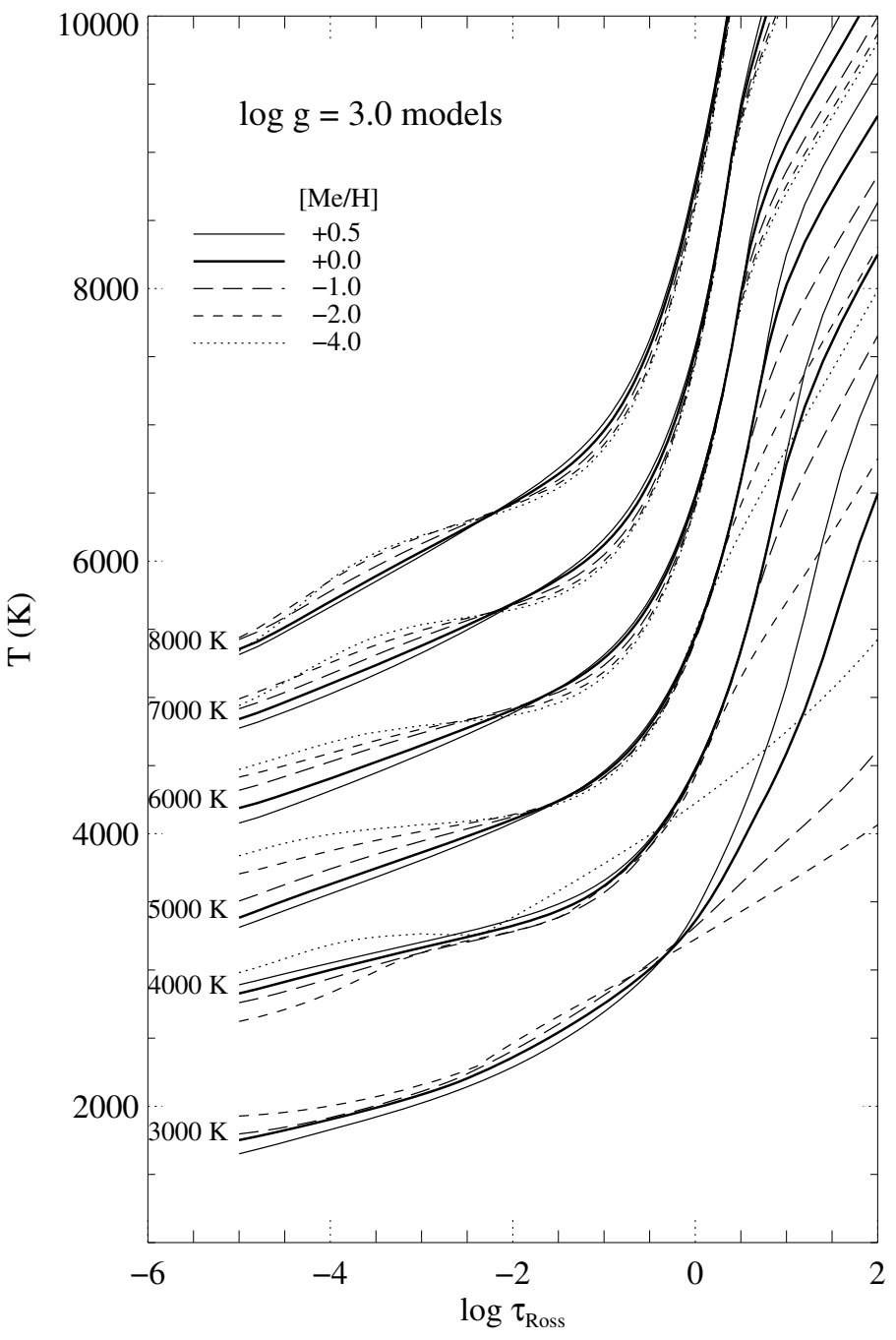

Fig. 2. The temperature structures for a set of model atmospheres with different $T_{\text {eff }}, \log g=3$ and different metallicities.

the coolest models with $\mathrm{H}_{2}$ convection zones at the surface as well as for models where the radiative pressure totally dominates the gas pressure.

\section{General properties of the models}

All models of the grid will be accessible via http://marcs . astro.uu.se/. The organization of the model files is described there. We give details of structures, thermodynamic variables, molecular partial pressures, and fluxes in $10^{5}$ wavelength points in the files. In Fig. 2, we present some sample temperature structures of grid models with different effective temperatures and metallicities. It is seen that the variation with metallicity at the surface and in the deep layers increases when $T_{\text {eff }}$ decreases from $8000 \mathrm{~K}$ to $5000 \mathrm{~K}$, and that this variation with metallicity changes sign at $4000 \mathrm{~K}$ in a non-trivial way. Subsequently, we will comment on this and other properties of the grid models. Our discussion here is confined to general properties of the model structures, while more details on models of particular types of stars, as well as discussion of model fluxes are deferred to later papers in this series. 


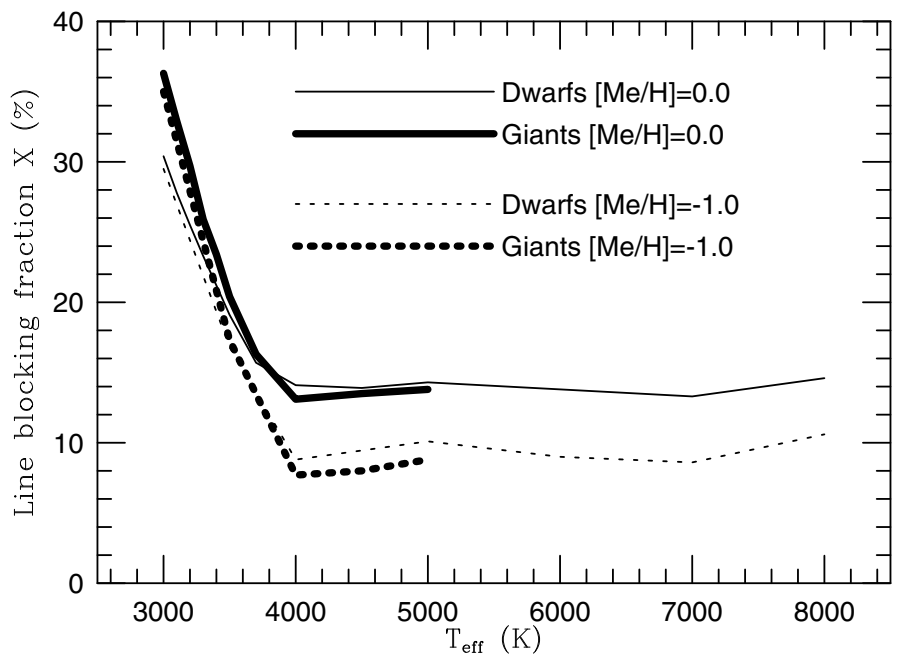

Fig. 3. The blocking fraction $X$ in percent for models in the grid with two different metallicities. The dwarf models all have $\log g=4.5$ while the giant models have $\log g$ values increasing with temperature, from $\log g=0.0$ at $T_{\text {eff }}=3000 \mathrm{~K}$ to $\log g=3.0$ at $T_{\text {eff }}=5000 \mathrm{~K}$.

\subsection{General effects of blanketing}

The effects of spectral lines on stellar atmospheres ("blanketing") have been explored thoroughly since it became possible to calculate models with lines included in the 1960s and 1970s. Here, we shall make a short summary of these results as a background for some more specific comments on the blanketing effects in the new grid.

In general, the blocking of radiation leads to heating of the deeper layers of the atmospheres ("back-warming"). Here and subsequently we measure the back-warming effect at a given continuum optical depth, in practice at $\lambda=500 \mathrm{~nm}$ (i.e., not $\tau_{\text {Ross }}$, which for the cooler models is strongly affected by line absorption). The back-warming is in fact easily estimated directly from the definition of the effective temperature by assuming that a model with effective temperature $T_{\text {eff }}$ is heated such that the deeper layers correspond to an unblanketed model with an effective temperature,

$T_{\mathrm{eff}}^{\prime}=(1-X)^{-\frac{1}{4}} \cdot T_{\mathrm{eff}}$,

where $X$ is the fraction of the integrated continuous flux blocked out by spectral lines,

$X=\frac{\int_{0}^{\infty}\left(F_{\text {cont }}-F_{\lambda}\right) \mathrm{d} \lambda}{\int_{0}^{\infty} F_{\text {cont }} \mathrm{d} \lambda}$.

The validity of this relation has been tested for models with different fundamental parameters by calculating $X$ of these models, and then we constructed unblanketed models with effective temperatures revised according to the relation. Next, we compared these models with the corresponding blanketed ones. The test came out favourably - for models all over the parameter space of the grid, the $T\left(\tau_{\text {Ross }}\right)$ structures in the interval $-1 \leq \tau_{\text {Ross }} \leq 0.5$ were reproduced astonishingly well by the corresponding unblanketed ones; the additional steepening of the temperature gradient across the $\tau_{\text {Ross }}$ interval for the blanketed model with solar abundances may typically amount to $0.02 \cdot T_{\text {eff }}$ per decade in $\tau_{\text {Ross }}$.

We also calculated the total blocking fraction $X$ for the grid models and find the remarkable behaviour shown in Fig. 3. Thus, $X$ stays nearly constant for the temperature interval

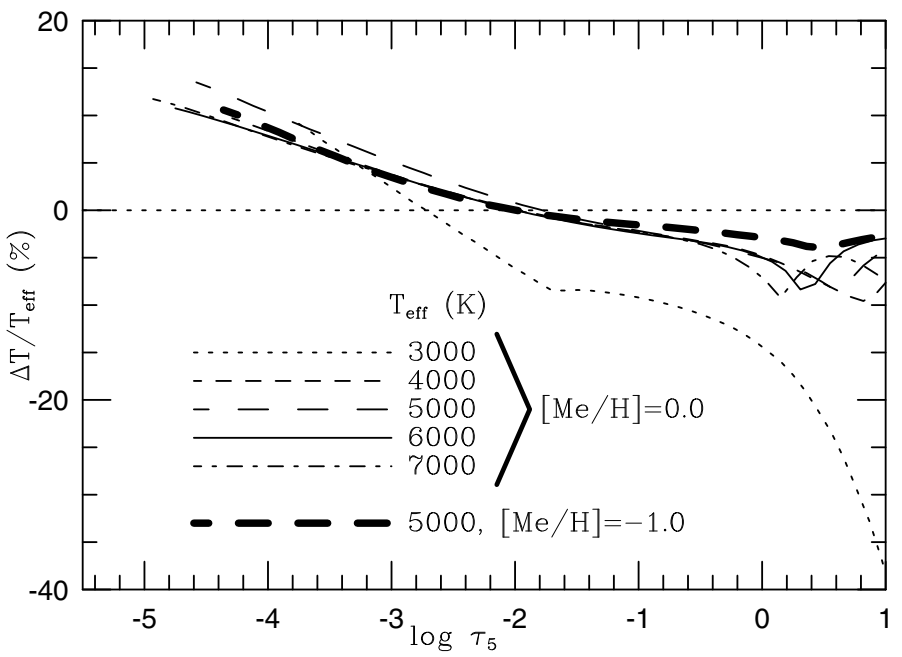

Fig. 4. The effects of switching off line absorption on the temperature structure of a sequence of models with $\log g=3.0$ and solar metallicity. Note that $\Delta T \equiv T$ (nolines) $-T$ (lines). It is seen that the blanketing effects are fairly independent of effective temperature for models with $T_{\text {eff }} \geq 4000$.

$8000 \mathrm{~K} \geq T_{\text {eff }} \geq 4000 \mathrm{~K}$ for a given metallicity, and is closely identical for dwarfs and giants, while below $4000 \mathrm{~K}$ it increases as temperature goes down, due to the heavy $\mathrm{TiO}$ and $\mathrm{H}_{2} \mathrm{O}$ blocking. Here, the metallicity sensitivity of $X$ decreases while the gravity sensitivity increases. The decreasing metallicity sensitivity reflects the fact that the molecular lines fill in the continuum regions in the spectrum and define the $\tau_{\text {Ross }}$ scale $\left(T\left(\tau_{\text {Ross }}\right)\right.$ being rather robust against metallicity changes).

For the upper layers of the atmospheres the situation is more complex, as was discussed, e.g., by Gustafsson et al. (1975); Gustafsson \& Olander (1979); and Carbon (1979); and as is illustrated in Fig. 4. In short, for LTE the spectral-line absorption may cause a cooling or a heating, depending on whether the absorption is located on the long-wavelength side of the local Planck function (cooling, Case 1) or on the short-wavelength side. In the latter case, heating (2a) or cooling (2b) may result, depending on whether the absorption is concentrated to the upper layers $(2 a)$ or whether it extends to the deeper atmosphere (2b). Examples of Case 1 are the cooling by the IR VR lines of CO for models of late G stars (cf. Johnson 1973; Gustafsson et al. 1975) and by $\mathrm{H}_{2} \mathrm{O}$ in the IR for later M stars. A clear example of Case $2 \mathrm{a}$ is the heating by the electronic bands of TiO in early M-star models (Krupp et al. 1978), while a Case $2 \mathrm{~b}$ example is provided by the metal-line absorption in the UV and blue for F and G stars. We note in passing that these surface effects are all dependent on the LTE assumption - in particular, if the spectral lines are partially formed in scattering processes the effects may be significantly smaller due to the weaker coupling of the radiation to the temperature of the local gas. The back-warming effects, on the other hand, are not strongly dependent on the assumed line-formation mechanism as long as the amount of blocking is unchanged. Missing or erroneous line absorption data may, however, still be of significance as a source of systematic errors in the back-warming effects.

The general effects of blanketing are illustrated for the grid models by a test where all line absorption was switched off. All models with $T_{\text {eff }} \geq 4000 \mathrm{~K}$ and solar metallicity show a similar response: the temperature is increased by about $10 \%$ in the outermost layers if the line opacity is neglected, an increase that gradually diminishes to zero at $\tau_{\text {Ross }}=0.01$ and then changes 


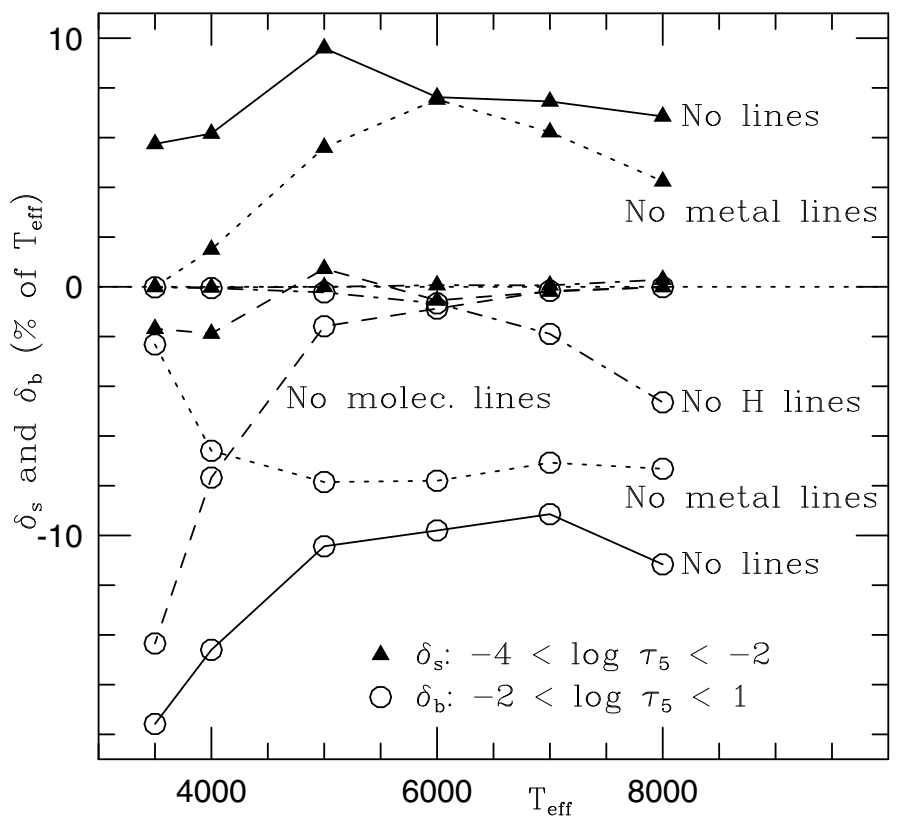

Fig. 5. Structural effects on the surface (black triangles) and depth (open circles) of model atmospheres by different groups of spectral lines. The solid lines show surface-heating and bottom-cooling as defined in Eq. (37) when all spectral lines are removed in the calculation of solarmetallicity dwarf models $(\log g=4.5)$. The dotted, dashed, and dashdotted lines show, respectively, the effects of removing only the metal lines, the molecular lines, or the hydrogen lines.

to a lowering of the temperatures at greater depth since backwarming is inhibited (cf., Fig. 4). For the coolest giants the effects become considerably greater.

We shall now give some more specific comments on effects of blanketing in the new grid, with remarks on the effects of various opacity sources and on the effects of changing abundances and microturbulence. Furthermore, we shall comment on the effects of sphericity and explore some interesting coupling between these latter effects and blanketing.

\subsection{Effects of different opacity sources}

In Fig. 5, we present the effects of different opacity sources, measured as integrated effects on the surface and at depth, in a set of models for main-sequence stars. We have thus defined the quantities

$\delta_{\mathrm{s}} \equiv \int_{-4}^{-2} \Delta T\left(\tau_{5}\right) \operatorname{dlog} \tau_{5} / \int_{-4}^{-2} \mathrm{~d} \log \tau_{5}$
$\delta_{b} \equiv \int_{-2}^{1} \Delta T\left(\tau_{5}\right) \operatorname{dlog} \tau_{5} / \int_{-2}^{1} \mathrm{~d} \log \tau_{5}$.

$\Delta T$ is the quantity

$\Delta T\left(\tau_{5}\right) \equiv\left[T\left(\tau_{5}\right)-T^{\prime}\left(\tau_{5}\right)\right] / T_{\mathrm{eff}}$

where $T\left(\tau_{5}\right)$ is the temperature structure measured at the continuum optical depth at $5000 \AA$ and $T^{\prime}\left(\tau_{5}\right)$ the corresponding quantity for a modified model atmosphere with the same fundamental parameters, but for which line opacity sources have been neglected individually or simultaneously (such that the corresponding model is unblanketed). The reason why $\tau_{5}$ and not $\tau_{\text {Ross }}$ were chosen here is that the Rosseland mean is directly affected by the spectral lines, in particular for the cooler stars, while we

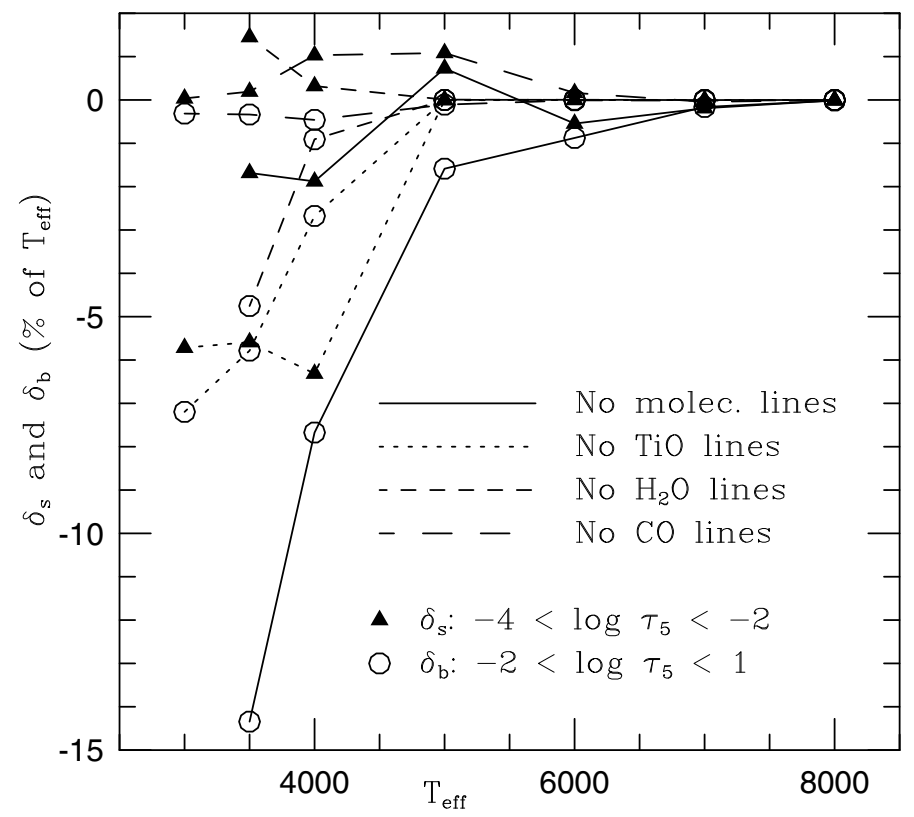

Fig. 6. Similar to Fig. 5. The structural effects of molecular lines separated for different species to show the results of removing the line opacity of $\mathrm{TiO}$, water, and $\mathrm{CO}$, respectively.

here wish to separate these effects on the temperature structure from those on the $\tau$ scale.

We see from Fig. 5 that for a sequence of main-sequence models with solar metallicity the total blanketing effect, both at the surface and in the deeper layers, is rather temperature independent. The structural effects of spectral lines are surprisingly similar for all solar-type stars. Thus, the mean surface cooling amounts to about $7 \%$ of $T_{\text {eff }}$ and the mean heating of the layers below $\tau_{5}=10^{-2}$ is about $11 \%$ over most of the effective-temperature interval. I.e., the somewhat decreasing importance of metal lines when proceeding towards the hot end of our effective-temperature interval is rather well compensated for by the increasing hydrogen-line blanketing. The diminishing effects of metals for the cooler models are compensated for by the molecular blanketing. These compensation effects explain why relatively old differential abundance analyses, made by primitive model atmospheres such as scaled solar models, are often found to agree rather well with more recent results based on models with much more complete atomic and molecular data. In Fig. 6, we display the corresponding effects when absorption by different molecular species is left out. It is seen that the only significant molecular effects at the surface for these models are the surface cooling due to $\mathrm{CO}$ for moderately cool models and to $\mathrm{H}_{2} \mathrm{O}$ for the coolest ones and the surface heating due to TiO, while in the deeper layers the significant back-warming is due to $\mathrm{TiO}$ and $\mathrm{H}_{2} \mathrm{O}$. Other molecules, like $\mathrm{CN}$, play only a very minor role in this respect.

\subsection{Effects of abundance changes}

The variation of certain abundances in the MARCS grid lead to effects on the models. Such effects were explored for the $\mathrm{CNO}$ abundances of $\mathrm{G}$ and $\mathrm{K}$ giant models by Gustafsson et al. (1975), for M and S stars by Plez et al. (2003) and for carbon stars by Lambert et al. (1986) and Jørgensen et al. (1992). The results given here are illustrative and complementary to those of previous studies - more detailed discussion of abundance effects will be presented in later papers in this series. 


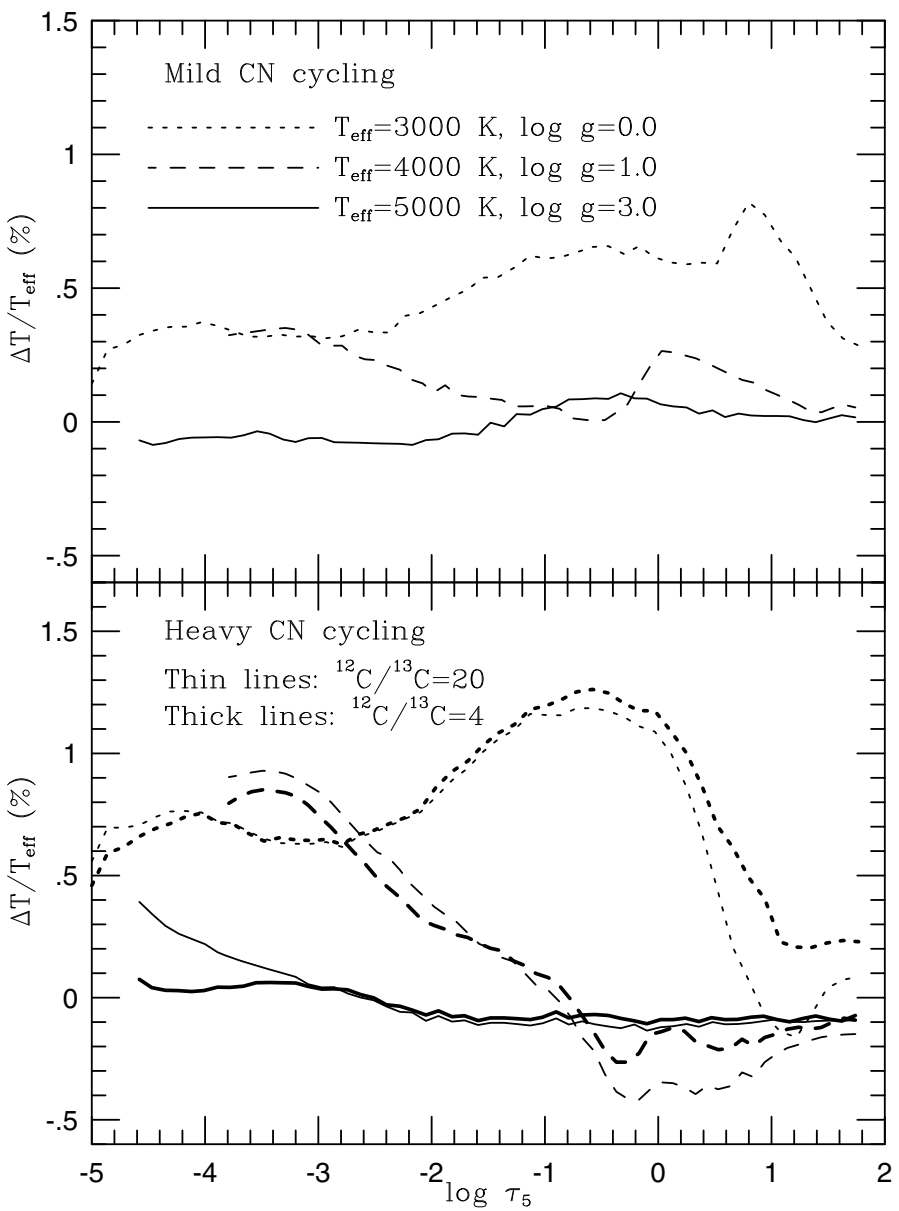

Fig. 7. The effects of dredge-up of $\mathrm{CN}$-cycled material to the surfaces of Pop I giant stars as illustrated by model atmospheres with different $\mathrm{C}$ and $\mathrm{N}$ abundances. In the top panel, carbon has been converted to nitrogen such that the $\mathrm{C} / \mathrm{N}$ abundance ratio has been lowered from the solar ratio of 4.07 to 1.5 , typical for the first dredge-up in low-mass stars. In the lower panel, the ratio has been further lowered to 0.5 and there also the effects of changing the ${ }^{12} \mathrm{C} /{ }^{13} \mathrm{C}$ ratio are displayed. The stronger back-warming of the $3000 \mathrm{~K}$ models is due to the increased importance of water as more oxygen gets available when the $\mathrm{CO}$ abundance is decreased by the reduction of carbon while the heating effect at the surface merely reflects the inadequacy of $\tau_{5}$ for this heavy line-blocked model - for a plot of the temperature vs. $\tau_{\text {Ross }}$, an increased cooling at the surface appears. In the $5000 \mathrm{~K}$ model, the decreased CO cooling leads to a hotter surface.

The recent considerable downward corrections of solar CNO abundances by Asplund and collaborators (Grevesse et al. 2007, and references therein) are found to lead to very minor effects for most regions in the parameter space spanned by the present grid. Thus, for the temperatures at given $\tau_{\text {Ross }}$, the effects are less than $1 \% \times T_{\text {eff }}$ for all depths and luminosities for giants at solar metallicity. The most pronounced effects in the surface layers appear in models with $T_{\text {eff }} \leq 3500 \mathrm{~K}$ and models with $T_{\text {eff }} \approx 5000 \mathrm{~K}$ where the reduced cooling due to $\mathrm{H}_{2} \mathrm{O}$ and $\mathrm{CO}$, respectively, is visible.

The CNO abundances in stars are modified through the first dredge-up along the subgiant-giant branch, mostly converting $\mathrm{C}$ to N (see, e.g., Boothroyd \& Sackmann 1999; Charbonnel 1994). The effects of this on the model atmospheres are, however, small or moderate, as illustrated in Fig. 7. The two most important effects are again the enhanced $\mathrm{H}_{2} \mathrm{O}$ surface cooling for the coolest models (since more $\mathrm{O}$ is available if $\mathrm{C}$ and thus $\mathrm{CO}$ is depleted),

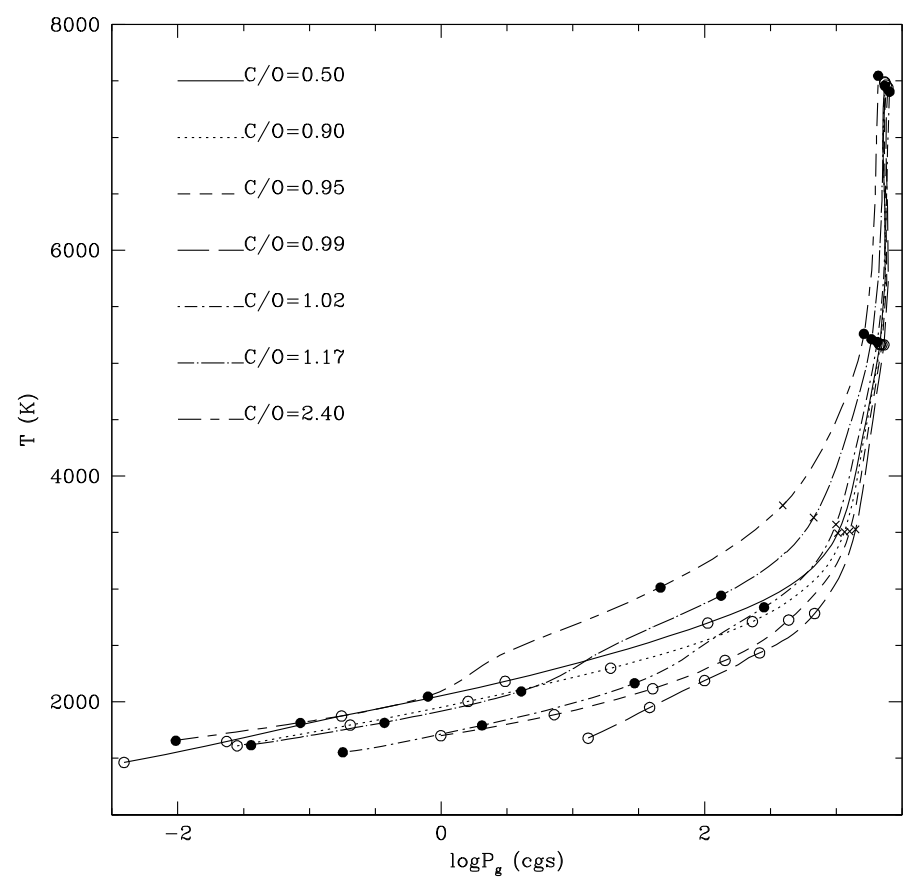

Fig. 8. A sequence of MARCS spherical model atmospheres with $T_{\text {eff }}=$ $3000 \mathrm{~K}, \log g=0.0, M=1 M_{\odot}$. The models have different abundance ratios, $\mathrm{C} / \mathrm{O}$, marked in the figure, but otherwise solar metal abundances. Points on the temperature-pressure relations with $\log \tau_{\text {Ross }}=-5,-4$, $-3,-2,-1,1$, and 2 are marked with circles, which are filled for the carbon-rich models $(\mathrm{C} / \mathrm{O} \geq 1.00)$. Points where $\log \tau_{\text {Ross }}=0$ are marked with $\times$. The figure demonstrates the strongly increasing pressures at given temperature and optical depth as the $\mathrm{C} / \mathrm{O}$ ratio increases, until $\mathrm{C} / \mathrm{O}$ reaches 1.0 when the situation is drastically reversed as the opacities increase and the pressures decrease again. The models with $\mathrm{C} / \mathrm{O} \leq 0.9$ show M-type model spectra, those in the interval $0.9 \leq 0.98$ $\mathrm{S}$-type spectra, while those with greater $\mathrm{C} / \mathrm{O}$ show $\mathrm{N}$-star spectra.

and a diminished $\mathrm{CO}$ cooling for the somewhat hotter models. The changes due to effects by $\mathrm{CN}$ are marginal. The accompanying changes of ${ }^{12} \mathrm{C} /{ }^{13} \mathrm{C}$ ratios are obviously only of minor significance.

The most severe atmospheric effects of CNO abundance changes occur, however, when the carbon abundance is raised by dredge-up on the asymptotic-giant branch and, as the carbon abundance approaches or exceeds that of oxygen, the star becomes an $\mathrm{S}$ or a $\mathrm{C}$ star, respectively, with drastic changes in radiation fields and thus temperature-pressure structure. This transition has been explored using MARCS models (for references, see above), and will be further discussed in Papers V and VI in the current series. As an illustration here, however, we present a sequence of models, with $\mathrm{C} / \mathrm{O}$ ratios ranging from 0.5 to 2.4 in Fig. 8.

The most metal-poor stars known until now show severe departures from "standard" CNO abundances: they seem to be comparatively very rich in CNO elements (cf., Christlieb et al. 2004; Frebel et al. 2005; Norris et al. 2007, and references therein). In general, there is a group of low-metallicity stars with considerably enriched carbon abundances, so-called carbon-enriched metal-poor (CEMP) stars, (see, e.g., Aoki et al. 2007), which may show carbon enrichments ranging in the interval between a factor of 10 and 1000 relative to a solar $\mathrm{C} / \mathrm{Fe}$, as well as similarly considerable $\mathrm{N}$ enrichments and possibly also $\mathrm{O}$ enrichments. The effects on the atmospheric structures from these enrichments are illustrated in Fig. 9 for one representative CEMP giant model. 


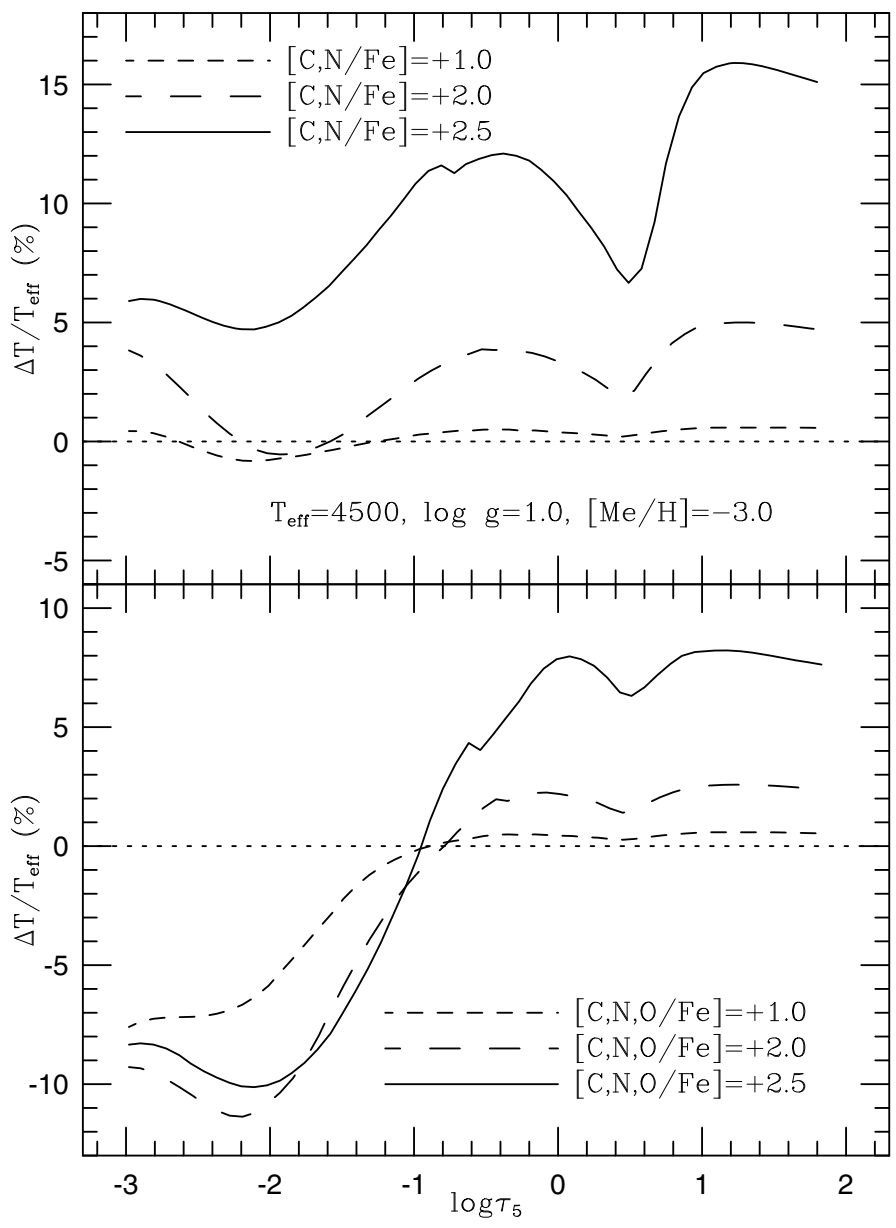

Fig. 9. The effects of non-solar $\mathrm{CNO}$ abundance ratios on extreme Pop II star models with $T_{\text {eff }}=4500 \mathrm{~K}, \log g=1.0,[\mathrm{Me} / \mathrm{H}]=-3.0$. In the top panel, the carbon and nitrogen abundances have been increased by factors of 10, 100, and 300 above the solar ratio to iron, oxygen and all other elements. The heating in the middle layers, primarily due to $\mathrm{C}_{2}$, is strong, and even more so at depth, which is due to the increased electron pressures and continuous opacities. In the lower panel, oxygen is also enhanced. The increased electron pressures in the more $\mathrm{CN}(\mathrm{O})$ rich models lowers the density and convection efficiency at a certain optical depth which increases the temperature gradient. The major surface cooling agent in the outer surface layers is $\mathrm{CO}$.

It is seen that the enhanced $\mathrm{C}$ and $\mathrm{N}$ abundances heat the layers $-2 \leq \log \tau_{5} \leq 0.5$ considerably for these model parameters (a result which was also found by Hill et al. 2000). Also, the deep layers get warmer, which is due to the increased electron contributions from the enhanced carbon. This increases the $\mathrm{H}^{-}$opacity, which lowers the density at a given optical depth and therefore reduces the convective energy flux, which increases the inner temperature gradient. These effects change if $\mathrm{O}$ is also enhanced correspondingly. Then the $\mathrm{CO}$ cooling takes over in the surface layers, and much of the $\mathrm{C}_{2}$ and $\mathrm{CN}$ heating absorption vanishes since most of the carbon is bound in the $\mathrm{CO}$ molecules.

The effects of the observed increase of $[\alpha / \mathrm{Fe}]$ for galactic, though seemingly not all, metal-poor stars can also be explored using the grid models. Although these changes are easily seen in spectra of the models, e.g., in the strengths of the $\mathrm{Mg}$ and $\mathrm{Ca}$ lines, the effects on the temperature-pressure structures are only minor. As is seen in Fig. 10, they are, however, of some significance for models with $T_{\text {eff }}<4500 \mathrm{~K}$ where the higher Ti abundance increases the surface heating as well as the backwarming of the $\mathrm{TiO}$ absorption. Simultaneously, the electron contributions from $\mathrm{Mg}$ and $\mathrm{Ca}$ increase and raise the $\mathrm{H}^{-}$opacity, which partly reduces the effects of the $\mathrm{Ti}$ abundance change. As discussed above for the CEMP star models, the higher electron pressures also reduce the densities at a given optical depth, which diminishes the convective flux and thus admits a stronger temperature gradient in the deep atmosphere.

It should finally be noted that systematic errors in the models, due to missing opacities, departures from LTE or convectively generated inhomogeneities and errors in calculated convective fluxes, may well vary systematically with chemical composition. Thus, the trends discussed in the present section may have to be revised when more realistic models are available.

\subsection{Blanketing effects of turbulence}

In the present grid, there are models calculated with different microturbulence parameters $\xi_{\mathrm{t}}$, but with otherwise identical input data. This makes it possible to systematically explore the dependence of the blanketing effects on microturbulence. Before discussing the results, we will present some considerations of what one could expect.

An increased microturbulence enhances the blocking of the spectrum by isolated saturated spectral lines, in proportion to $\xi_{\mathrm{t}}$. So, if all lines were saturated, one might estimate from Eq. (35) that the back-warming $\Delta T / T$ would increase by about $1 / 4$. $\Delta \xi_{\mathrm{t}} / \xi_{\mathrm{t}} \cdot X$. However, this is an upper limit since a considerable fraction of the spectral lines (though not necessarily those that contribute most significantly to the blocking) will not be saturated - their equivalent widths will be unaffected by a changing $\xi_{\mathrm{t}}$ and thus the total blocking is not changed. Also, for more metal-rich stars the overlapping of strong lines reduces the differential effects of a $\xi_{\mathrm{t}}$ increase as does the fact that many of these lines have strong damping wings. We thus expect the effects of microturbulence changes on the back-warming to be small for the most metal-poor stars where even the strongest lines are unsaturated, then increase but finally level off for the most metal-rich and coolest models, and never reach the estimated upper limit. These expectations are verified by the models, as is illustrated in Fig. 11. The differential back-warming caused by an increased microturbulence parameter, e.g., by a factor of 2 , is of about the same magnitude as the result of doubling the metallicity. This suggests that the total blocking contribution of lines on the flat part of the curve-of-growth (mainly sensitive to $\xi_{\mathrm{t}}$ ) is of similar significance as that of weak spectral lines (proportional in strength to the metallicity); this picture is complicated by both the effects of lines on the dampingpart of the curve-of-growth (with only a square-root dependence on abundance) and the strong abundance sensitivity of some diatomic molecules. With regard to the differential blanketing effects in the upper atmosphere when the microturbulence parameter is changed, the situation is not quite obvious. The first, heating, part of the radiation equilibrium integral in Eq. (30) will not change in itself if only the line-absorption profiles become broader. If the effects on $J_{\lambda}$ are taken into consideration, the decrease of the intensity over larger wavelength regions by the widening of the lines will, as long as they are weak, be compensated for by the increase of $J_{\lambda}$ in the line centre. However, for saturated lines, $J_{\lambda}$ will be reduced over broader wavelength intervals when $\xi_{\mathrm{t}}$ is increased, and thus the heating term will become smaller. The second, cooling, term will not change from a broadening of the line profiles. Thus, a net cooling is expected to occur. We see from Fig. 11 that, in practice, increasing $\xi_{\mathrm{t}}$ from 1 to $2 \mathrm{~km} \mathrm{~s}^{-1}$ leads to some moderate cooling (on the order of $0.5 \%$ 


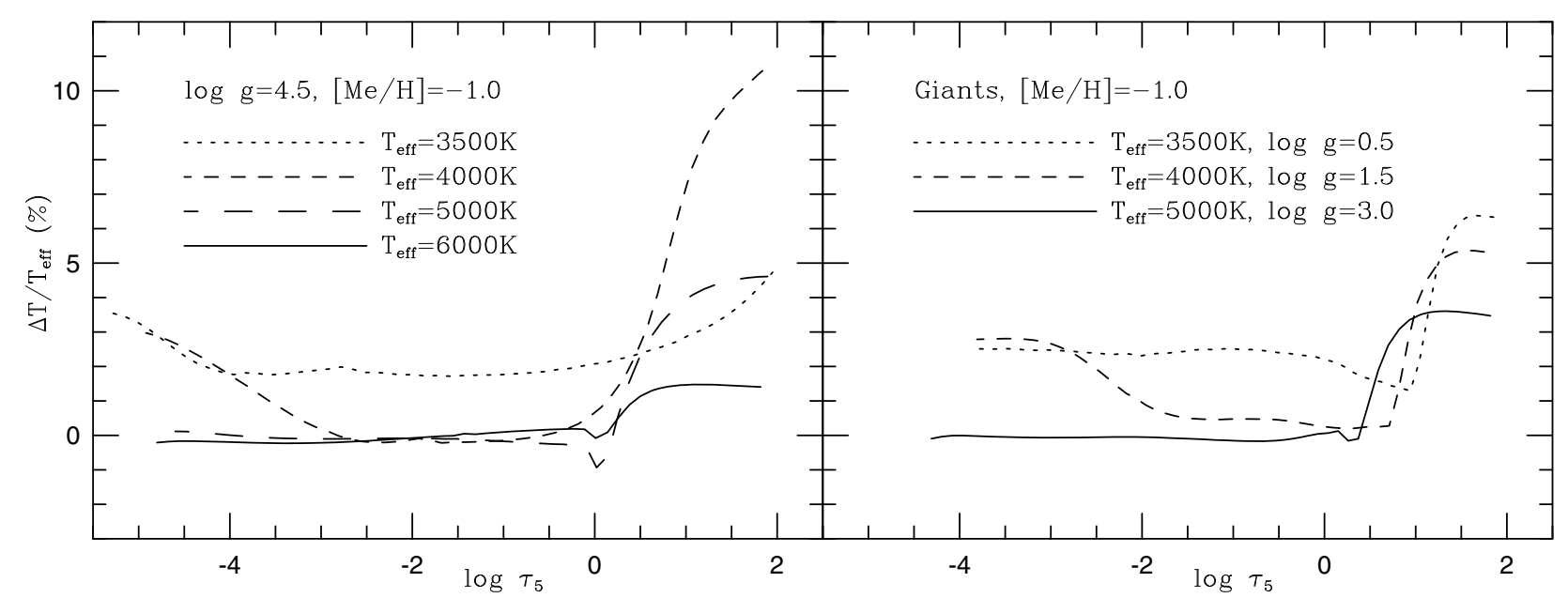

Fig. 10. The effects of an increase in the $\alpha$-element abundances by +0.4 dex on the temperature structure of models of dwarfs and giants with $[\mathrm{Fe} / \mathrm{H}]=-1.0$. The comparison model in each case is a corresponding model with no $\alpha$-element enhancement $([\alpha / \mathrm{Fe}]=0.0)$. The increased electron pressure lowers the density and convection efficiency at a certain Rosseland optical depth, which increases the temperature gradient in the deeper parts of a model.

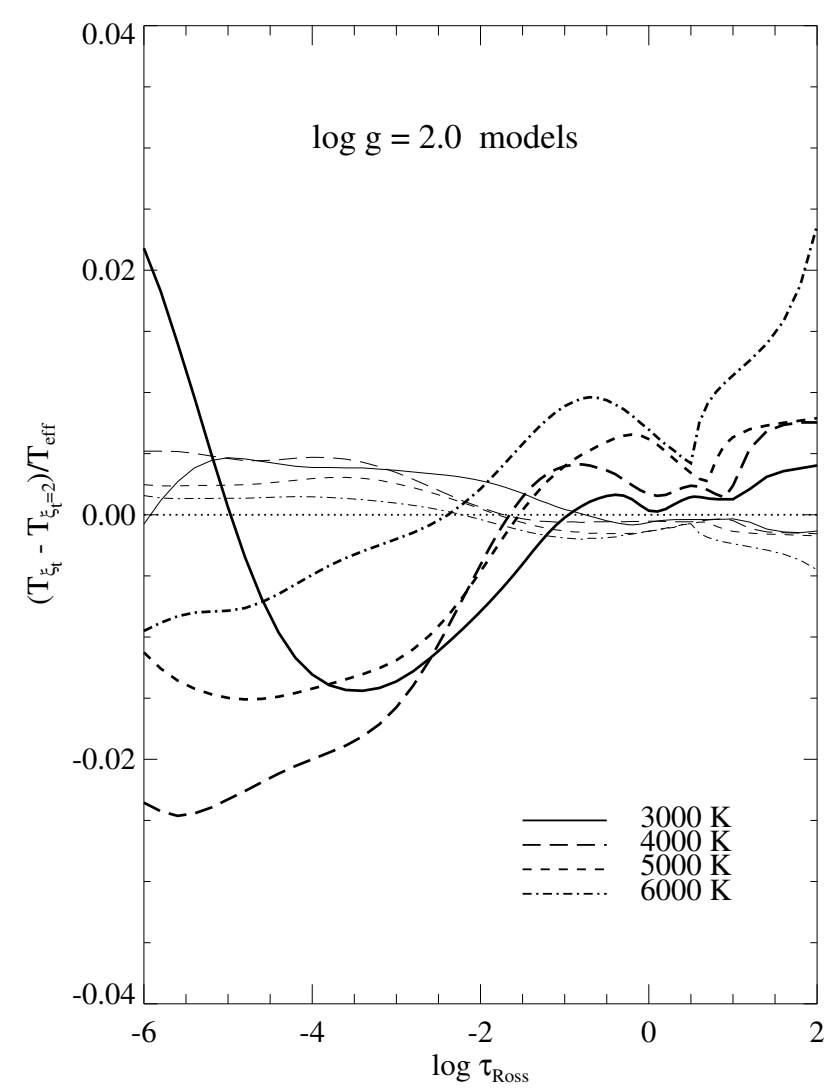

Fig. 11. $T\left(\tau_{\text {Ross }}\right)$ for models with different microturbulence parameters $\xi_{\mathrm{t}}$, minus $T\left(\tau_{\text {Ross }}\right)$ for a model with $\xi_{\mathrm{t}}=2 \mathrm{~km} \mathrm{~s}^{-1}$, for solar composition, $\log g=2.0$ and different effective temperatures. Thick lines denote models with $\xi_{\mathrm{t}}=10 \mathrm{~km} \mathrm{~s}^{-1}$, thin lines with $\xi_{\mathrm{t}}=1 \mathrm{~km} \mathrm{~s}^{-1}$.

in $T / T_{\text {eff }}$ ) of the upper layers for all effective temperatures, while further increase up to $10 \mathrm{~km} \mathrm{~s}^{-1}$ leads to further cooling (by up to $2 \%$, for the coolest models), however, this balance shifts to heating in the very surface layers.

Changes in the macroturbulent parameter (classically representing velocity shifts on geometrical scales corresponding to at least one optical depth unit in the continuum) are not able to change the model structures through radiative field changes. See
Sect. 3 above concerning effects of changes in the turbulent pressure, which may be related to both macro- and microturbulent motions.

Real stellar atmospheres are not believed to be well represented by the concepts of micro- and macroturbulence. Velocity gradients on intermediate scales are of vital importance, as has been shown by convective simulations (Nordlund \& Dravins 1990; Asplund et al. 2000a), and global gradients caused by pulsations as demonstrated in Lederer et al. (2006) and references therein. With respect to the effects on the radiative field, these gradients allow for more heating in spectral lines since the gas high up in the atmosphere may absorb hot radiation from lower layers that was not absorbed below; similarly, the gas may cool more efficiently through spectral lines since the shifts make the gas more transparent in overlying layers. However, even more important are the hydrodynamical effects due to gas expansion and compression on the energy balance. Altogether, this makes the representation of the energy balance in real stars by classical models quite problematic; also, there is no hope to gain further insight into the physics of these systems by fine-tuning the turbulence parameters.

\subsection{Effects of sphericity}

The properties of spherically-symmetric model atmospheres for late-type stars have been discussed or commented upon in numerous studies. The area was first pioneered by Schmid-Burgk \& Scholz (1975) and Schmid-Burgk et al. (1981, and papers cited therein); and later explored by Plez (1990), Plez et al. (1992); Jørgensen et al. (1992); Hauschildt et al. (1999); and Heiter \& Eriksson (2006). The papers by Plez and collaborators, by Jørgensen and collaborators, and by Heiter \& Eriksson, all build on earlier versions of the MARCS code. These papers demonstrate that the effects of sphericity are in general important for the temperature structures, causing relative temperature effects on the order of $1 \%$ or more, for extensions of the atmosphere normalised on the stellar radius greater than about $2 \%$. The relative extensions scale roughly as $g^{-1 / 2}$; the sphericity effects are thus mainly important for low-gravity models. These papers do not, however, systematically explore the effects on the models and fluxes of changing from plane-parallel to spherical 


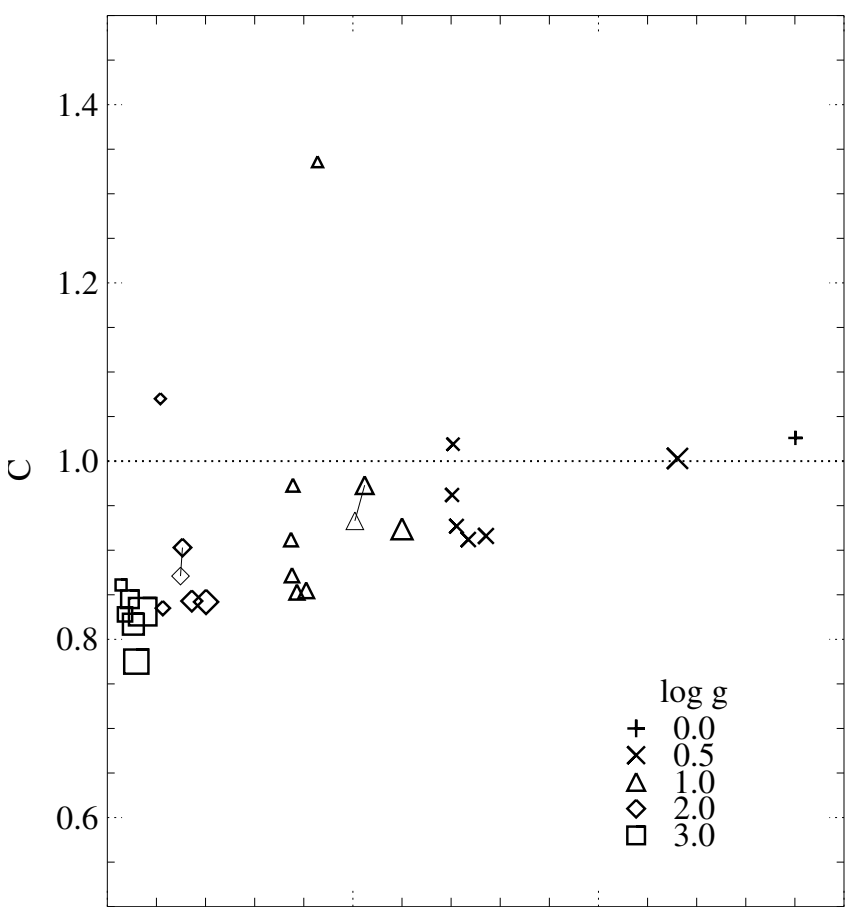

Fig. 12. The value of $C$ as calculated from Eq. (40) with model values of $z_{0} / R_{1}$ for a set of models with $T_{\text {eff }}$ ranging from $3000 \mathrm{~K}$ to $8000 \mathrm{~K}$ (with bigger symbols the higher the effective temperature) and metallicities $[\mathrm{Me} / \mathrm{H}]=0.0$, except for two models with $[\mathrm{Me} / \mathrm{H}]=-1.0$, which are attached with thin lines to corresponding $[\mathrm{Me} / \mathrm{H}]=0.0$ models. $\eta$ was chosen $=1$. The value of $C$ when based on the value of the extension $z_{0} / R_{1}$ estimated from Eq. (39) is 1.0 and shown by the horizontal dotted line.

symmetric geometry. Here, some further comments will be made on this topic on the basis of the new model grid.

The extension $z_{0}$ of the stellar atmosphere, measured as the geometrical thickness of the atmosphere (for instance, defined as the layers with $\left.-5.0 \leq \log \tau_{\text {Ross }} \leq 0.0\right)$ is a key factor in the discussions. It is easy to estimate the extension from the hydrostatic-equilibrium equation, the definition of optical depth and the gas law for an ideal gas. The relative thickness of the atmosphere from optical depth $\tau$ up toward the surface at $\tau_{0}$ is thus:

$\frac{z_{0}}{R_{1}}=\frac{\mathcal{R}\langle T\rangle}{\left\langle\mu_{\mathrm{mol}}\right\rangle g R_{1}(\eta+1)} \cdot \ln \left(\frac{\tau}{\tau_{0}}\right)=4 \times 10^{-7} \frac{R_{1}^{\odot} \cdot T_{\mathrm{eff}}}{M^{\odot} \cdot(\eta+1)}$.

Here, we have assumed the variation of the pressure in the atmosphere to be dominated by the density variation - the temperature and the mean molecular weight $\mu_{\text {mol }}$ in the gas law were thus approximated by a constant characteristic temperature and molecular weight, $\langle T\rangle$ and $\left\langle\mu_{\mathrm{mol}}\right\rangle$ (here set $=1.26$ ), respectively. The mass absorption coefficient (opacity per gram) $\kappa_{\mathrm{g}}$ is assumed to vary (only) with the density $\rho, \kappa_{\mathrm{g}}=k \cdot \rho^{\eta}$. The model radius and mass are $R_{1}^{\odot}$ and $M^{\odot}$, respectively, in solar units.

According to our analytical estimate of the relative extension in Eq. (39), the quantity

$C=2.5 \times 10^{6}\left[\frac{z_{0}}{R_{1}}\right] \cdot \frac{M^{\odot}}{R_{1}^{\odot} \cdot T_{\mathrm{eff}}} \cdot(\eta+1)$

should be approximately constant and equal to 1.0. It is seen in Fig. 12 that the relative extension is fairly well accounted for by the approximate expression, and that the choice of $\eta=1$ is a reasonable fit for an extensive part of the HR diagram, which is

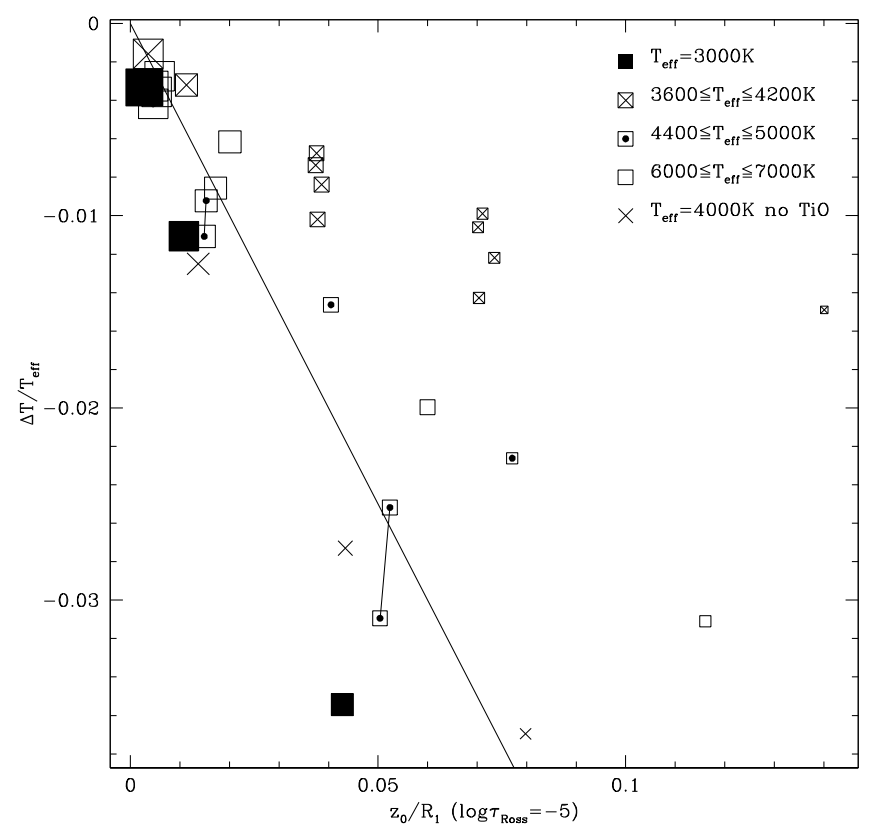

Fig. 13. The difference in the surface temperature (at $\log \tau_{\text {Ross }}=-5$ ) between spherical and plane-parallel models with $T_{\text {eff }}$ ranging from $3000 \mathrm{~K}$ to $7000 \mathrm{~K}$ (with bigger symbols the higher $\log g$ ) and metallicities $[\mathrm{Me} / \mathrm{H}]=0.0$, except for two models with $[\mathrm{Me} / \mathrm{H}]=-1.0$, which are attached with thin lines to corresponding $[\mathrm{Me} / \mathrm{H}]=0.0$ models (the lower symbols in the pairs representing the more metal-poor models). Three models at $T_{\text {eff }}=4000 \mathrm{~K}$ have also been calculated with TiO absorption neglected and are denoted by crosses - it is seen that the positions of these models depart very significantly from the corresponding standard models. The relation according to Eq. (41) is shown as a straight line.

to be expected since the dominating $\mathrm{H}^{-}$absorption per gram is roughly proportional to the pressure. Note that the relative extension of an atmosphere is relatively independent of the magnitude of the opacity, which cancels in the zero-order approximation. A depth-variation of $\kappa$ is of some significance, but only marginally so. The most important sphericity effects related to the opacity are more indirect, through the effects that a changed temperature structure may lead to, in particular when molecular absorption is switched on, as will be demonstrated below.

We now turn to the sphericity effects on the temperature structure of the models. The temperature in radiative equilibrium is set by the radiative field, following Eq. (30). The basic effects due to sphericity at a point high up in a stellar atmosphere come from the general dilution of the radiative field, partially due to the absence of incoming radiation from angles $\mu \leq \mu_{0}$ where $\mu_{0}$ gradually increases the further from the optically-thick layers that the point is situated. Basically, this leads to less radiative heating of the upper layers of the atmosphere and thus leads to cooler temperatures there. One may prove that this reduction of the mean intensity for small extensions will scale with $\left(z_{0} / R_{1}\right)^{1 / 2}$. For larger distances from the star, a simple and approximative estimate for the temperature effect, was presented by Gustafsson et al. (1975; see also Böhm-Vitense 1972) who assumed that the radiative flux should be roughly proportional to radius $r^{-2}$ and to temperature $T^{4}$ as one may estimate from Stefan-Boltzmann's law. This leads to the estimate

$\Delta T / T \approx-0.5 \times z_{0} / R_{1}$.

The effects of sphericity on the temperature structures of the models are shown in Fig. 13 and compared with the approximate 


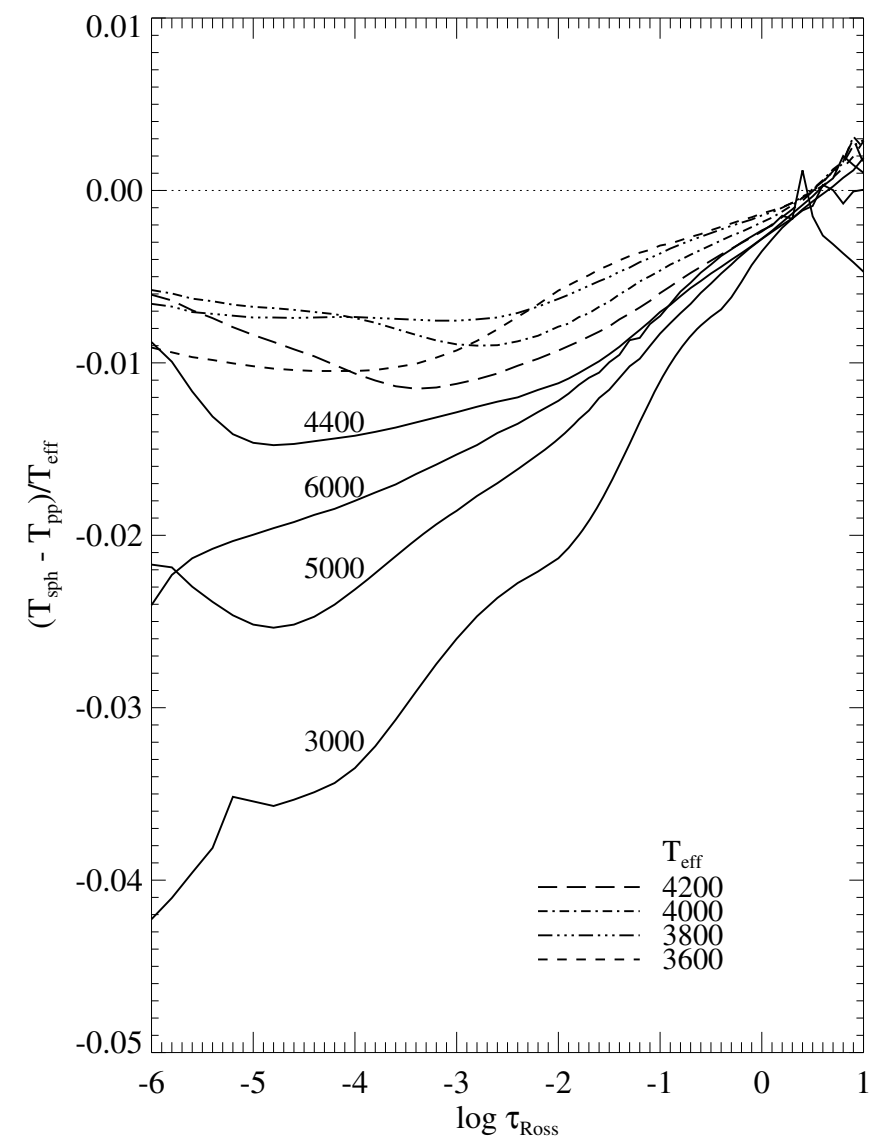

Fig. 14. The difference between temperature structures of spherically symmetric and corresponding plane-parallel models with $\log g=1.0$, $M=1.0 M_{\odot},[\mathrm{Me} / \mathrm{H}]=0.0$, and $T_{\text {eff }}$ ranging from $3000 \mathrm{~K}$ to $6000 \mathrm{~K}$. The complex variation of the sphericity effects in the upper layers when TiO sets in as a heating agent at $T_{\text {eff }} \approx 4000 \mathrm{~K}$ is illustrated.

expression in Eq. (41). The agreement is satisfactory, though certainly not perfect, which is partially explained by the approximations made in deriving it. As important are the effects of the wavelength dependence of $\kappa_{\lambda}$, which gives the mean intensity $J_{\lambda}$ and the Planck function $B_{\lambda}$ in the radiation equilibrium integrals of Eq. (30) different weights for different models, depending in particular on the molecular absorption and resulting in the large scatter in Fig. 13.

Now turning to the detailed model results, the sphericity effects on $\Delta T / T$ are shown in Fig. 14 as a function of $\tau_{\text {Ross }}$ for a sequence of supergiant models with different effective temperatures. Obviously, the effects shift with $T_{\text {eff }}$. First being relatively small for the hottest models, they considerably increase for $T_{\text {eff }} \sim 5000 \mathrm{~K}$, then diminish again to reach a minimum at about $3800 \mathrm{~K}$ and finally again increase strongly as $T_{\text {eff }}$ goes towards $3000 \mathrm{~K}$. This behaviour may be interpreted as a coupling between the sphericity effects and the effects of molecular blanketing. The coupling may show up as positive or negative feedback. Sphericity cools the upper layers, relative to a plane-parallel model, and the molecular formation increases. Around $5000 \mathrm{~K}$ this leads to a strong surface cooling by CO. For cooler models $\mathrm{TiO}$ forms, which conversely heats the upper atmosphere. Finally, $\mathrm{H}_{2} \mathrm{O}$ takes over as a dominating opacity source, and that again cools the model surface. That this explains the behaviour can be tested by blocking out different molecular opacities from the model calculations. In Fig. 15 we thus show a sequence of models at $T_{\text {eff }}=4000 \mathrm{~K}$ with different $\log g$ and

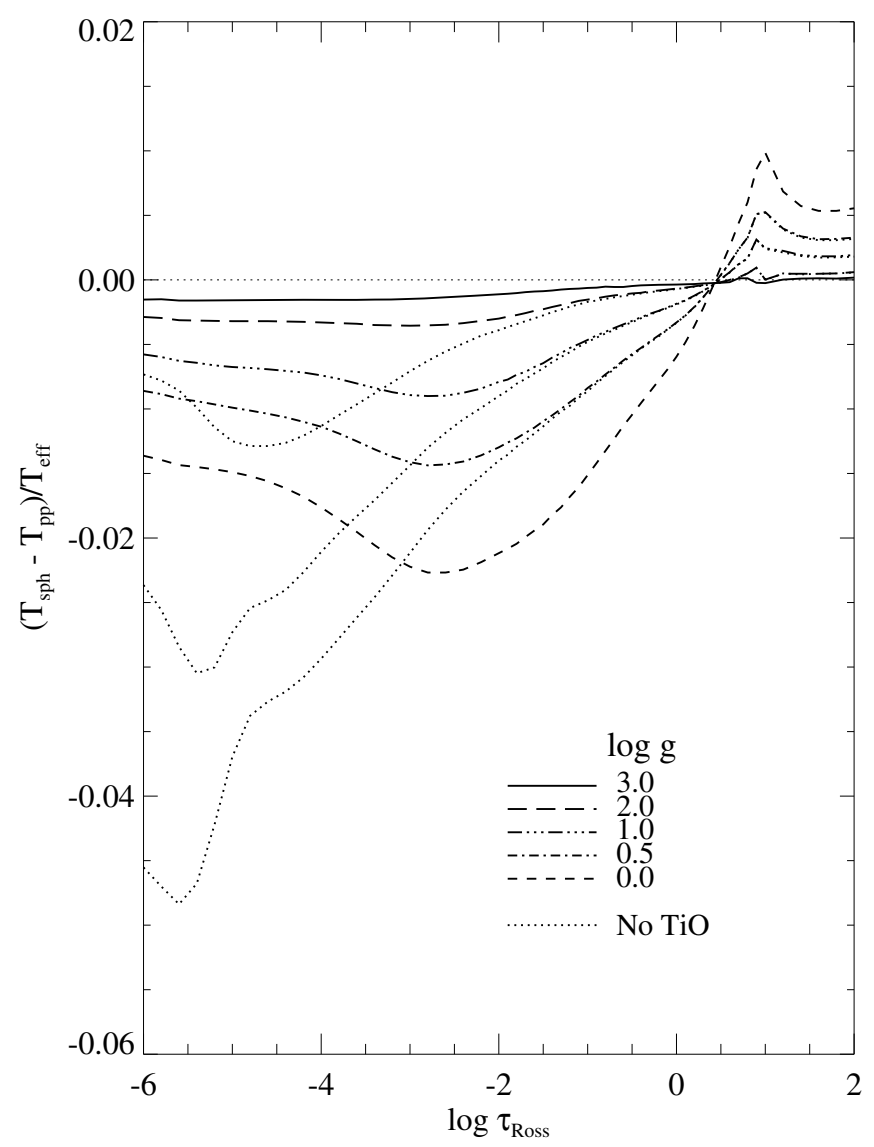

Fig. 15. The difference between temperature structures of sphericallysymmetric and corresponding plane-parallel models with $T_{\text {eff }}=4000 \mathrm{~K}$ and different $\log g . M=1.0 M_{\odot}$ and $[\mathrm{Me} / \mathrm{H}]=0.0$, and with the $\mathrm{TiO}$ absorption alternatively switched on and off (dashed lines, the gravities of these can be traced via the models to which they adhere at depth). The complex variation of the sphericity effects in the upper layers due to $\mathrm{TiO}$ is illustrated.

with and without TiO. It is clear that the differential sphericity effects above $\tau_{\text {Ross }}$ are changed significantly by the TiO absorption. From this figure we also see that the sphericity effects in the temperature structure are significantly greater than $1 \%$ for models with $\log g \leq 1.0$. However, if turbulent pressures are taken into account this latter value may be higher, according to Eq. (7).

\section{Comparison with other model grids}

We now compare MARCS grid models with the models of other existing grids, but confine this comparison to the temperature structures. Some additional comments on calculated fluxes will be be made in subsequent papers of this series.

The most extensive and widely-used grid of models for most of our parameter space are the plane-parallel models of Kurucz and collaborators (available on http://kurucz. harvard.edu). Among the different sets of models published there, it is particularly relevant to compare our models with those calculated by Castelli \& Kurucz (2003) with a standard mixinglength theory (without "convective overshoot"), which also seem to produce more consistent model parameters when applied to real data (Castelli et al. 1997). Some sample comparisons of model structures are shown in Figs. 16-18. It is seen that the agreement in the temperature structures is almost perfect for the models of solar-type dwarf stars of different metallicities. This 


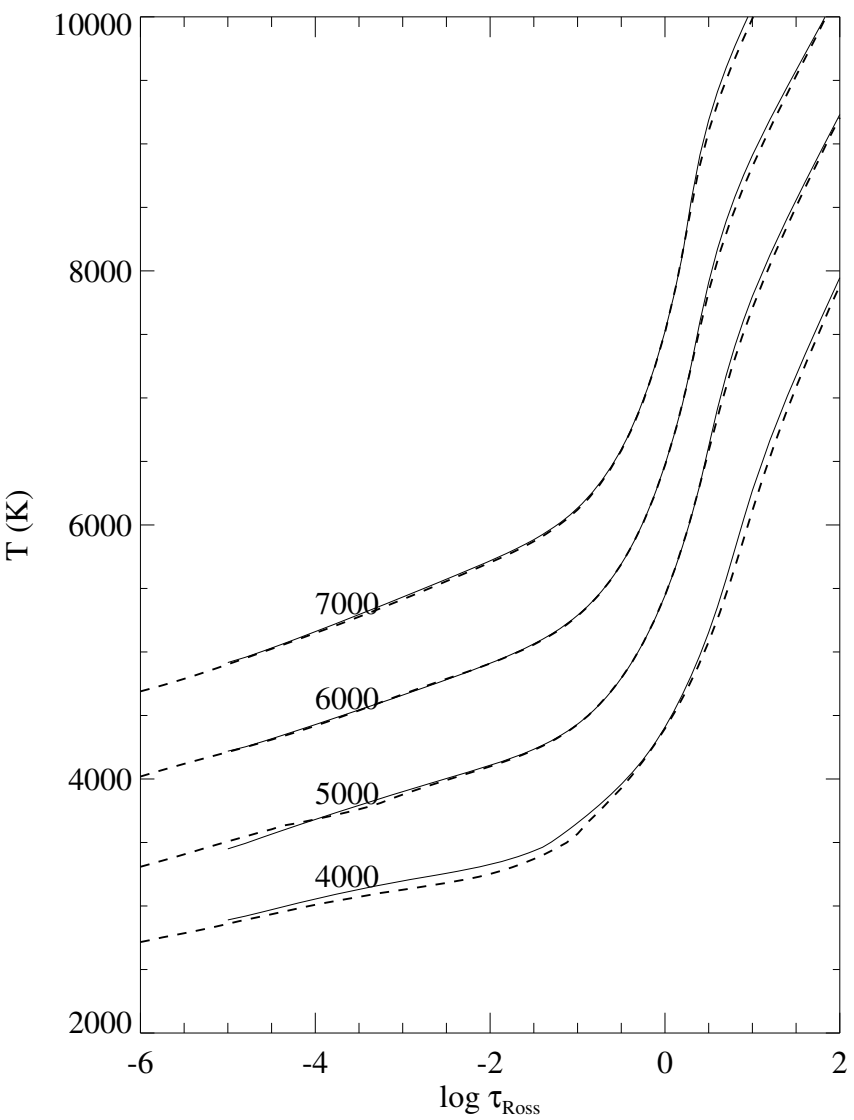

Fig. 16. MARCS model atmospheres (solid) for dwarf stars with $\log g=$ $4.5,[\mathrm{Me} / \mathrm{H}]=0.0$ and corresponding models from the Castelli \& Kurucz ODFNEW grid (dashed). The curves are labelled with relevant values of $T_{\text {eff. }}$.

is also true for models of early $\mathrm{M}$ dwarfs. For the models of giants and supergiants, the agreement is again good, although the MARCS models tend to be some $10-80 \mathrm{~K}$ cooler in the surface layers where $\tau_{\text {Ross }} \leq 10^{-2}$. The agreement is also very satisfactory if pressures or densities are intercompared. In view of the fact that these two grids of models are made with two totally independent numerical methods and computer codes, with independent choices of basic data (although Kurucz's extensive lists of atomic line transitions are key data underlying both grids), this overall agreement is both satisfactory and gratifying.

The situation was somewhat less satisfactory for models from the NextGen grid of spherically-symmetric models, calculated with the PHOENIX code (Hauschildt et al. 1999, models obtained by private communication in 1996). In the inner photospheres the agreement is good, but again a difference systematically appears at the surface, starting around $\tau_{\text {Ross }} \approx 10^{-2}$, with the MARCS models being cooler, but now by as much as typically $250 \mathrm{~K}$. (As is seen in Fig. 19, the agreement between the Castelli \& Kurucz and the MARCS models is generally much better than with the NextGen models.) However, more recent LTE models (PHOENIX-ACES-2008) obtained by Hauschildt with an updated version of the PHOENIX code (Hauschildt, private communication) and which are also based on Kurucz line data, agree very well with the MARCS models. The basic reason for this improvement in the fit seems to be due to changes in molecular opacities. Again, the present overall fit is very satisfactory in view of the independence of the methods of the MARCS and PHOENIX teams.

A number of comparisons with models made by previous versions of MARCS have also been performed. In Fig. 20, we

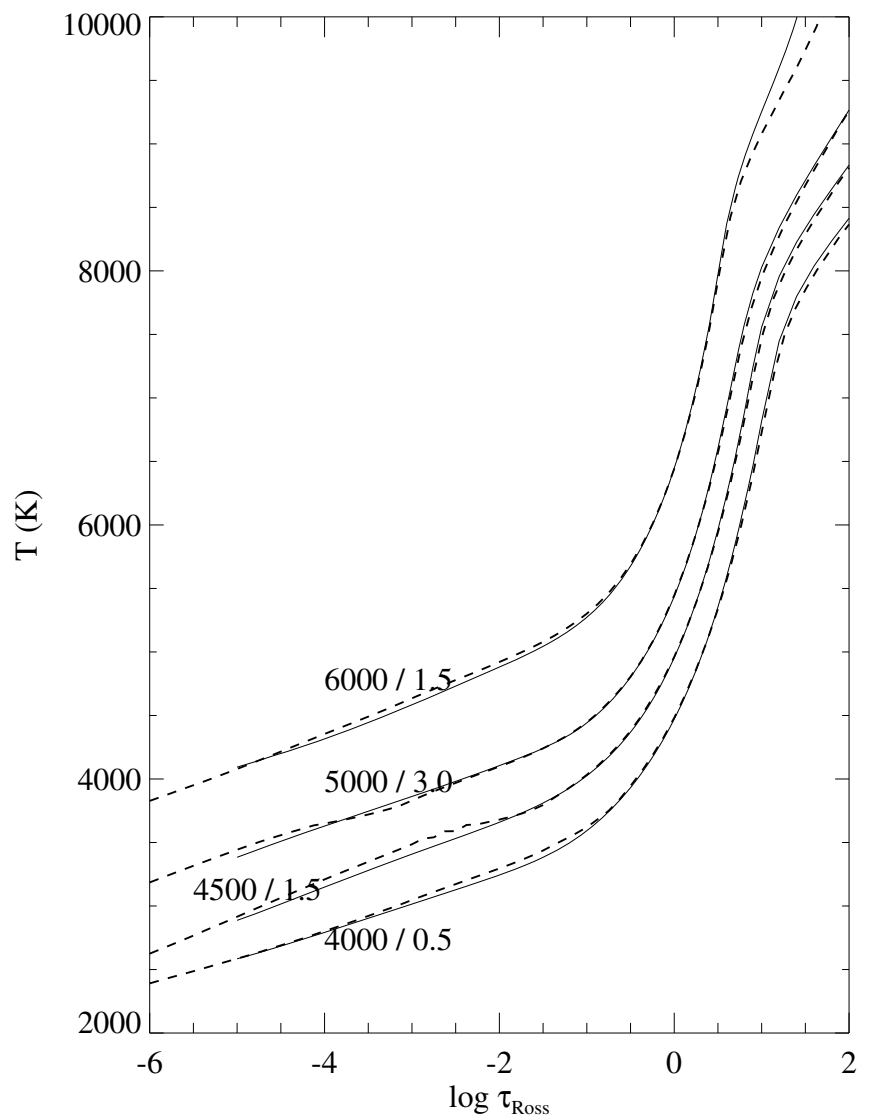

Fig. 17. MARCS model atmospheres for giants and supergiants with $[\mathrm{Me} / \mathrm{H}]=0.0$ ( solid) and corresponding models from the recent Castelli $\&$ Kurucz grid (dashed). The curves are labelled with relevant values of $T_{\text {eff }}$ and $\log g$.

thus compare with models of G and K giants (Bell et al. 1976) calculated with the first version of MARCS. It is seen that the present temperature structures agree quite well with the older ones in spite of the much more incomplete line absorption data of those models. (An exception from this is seen in the outer layers of the $4000 \mathrm{~K}$ model, where the TiO absorption, missing in the BGEN models, contributes a surface heating of the more recent model). The pressures in the present models are, however, significantly lower, which reflects their more heavy opacities. The good agreement in the temperature structures must thus, at least partially, be fortuitous, e.g., reflecting that the line absorption missing in the 1976 models is not heavily biassed towards the ultraviolet or the red spectral regions relative to the absorption that is now included. In Fig. 20, we also show one example where a spherically-symmetric model for a late-type M supergiant is compared with a previous spherically-symmetric MARCS model of Plez et al. (1992). The considerable differences in the upper photosphere are due to the new and improved molecular data used now and its more detailed representation, in particular, for the $\mathrm{H}_{2} \mathrm{O}$ absorption.

\section{Interpolation between the models}

It is useful to be able to interpolate the model structures to a set of parameters $T_{\text {eff }}, \log g$, and $[\mathrm{Me} / \mathrm{H}]$ different from those tabulated in the grid. Such an interpolation is not completely straightforward, as strong non-linearities appear in the behaviour of some of the thermodynamic variables when stellar parameters are varied. One program was developed by Masseron (2006) 


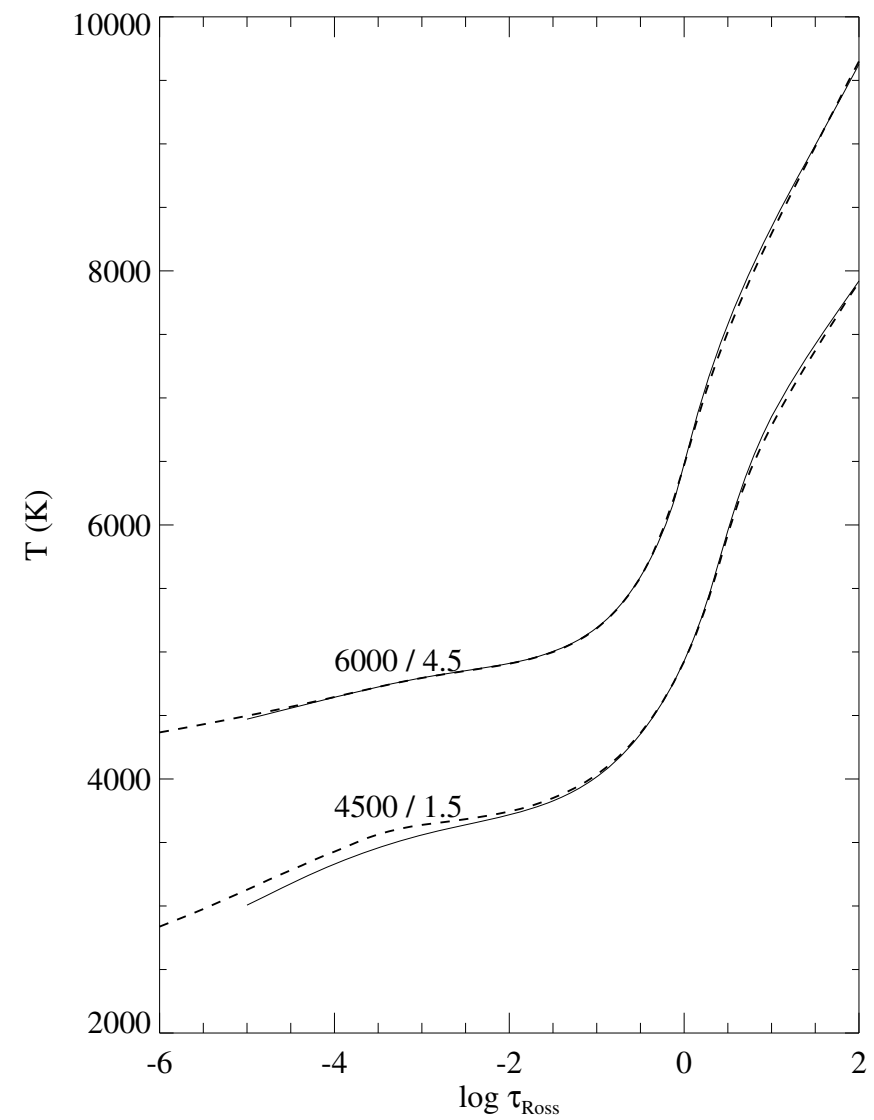

Fig. 18. MARCS model atmospheres with $[\mathrm{Me} / \mathrm{H}]=-2.0$ (solid) and corresponding models from the Castelli \& Kurucz ODFNEW grid (dashed). The curves are labelled with relevant values of $T_{\text {eff }}$ and $\log g$.

and extensively tested by him on a previous grid of MARCS models encompassing the following range of parameters: $3800 \mathrm{~K}<T_{\text {eff }}<7000 \mathrm{~K}, 0.0<\log g<5.0,-4.0<$ $[\mathrm{Me} / \mathrm{H}]<0.0$. It interpolates $T, P_{\mathrm{g}}, P_{\mathrm{e}}, \kappa_{\text {Ross }}$, and the geometrical depth as a function of $\tau_{\text {Ross }}$. The interpolated model must lie inside a complete cube of existing models in the parameter space $\left(T_{\text {eff }}, \log g,[\mathrm{Me} / \mathrm{H}]\right)$, and the interpolation is optimized to account for non-linearities in the grid. With the actual grid parameter steps, maximum errors in the interpolated quantities should remain below $0.25 \%$ for $T$ and a few $\%$ for $P_{\mathrm{g}}$ and $P_{\mathrm{e}}$, whereas $\kappa_{\text {Ross }}$ and the column density (rhox) are more difficult to interpolate. The program, with a detailed user manual, is available on the MARCS web site: http://marcs.astro.uu.se. An interpolation routine, applied to structures, as well as fluxes and colours of models by Kurucz and collaborators, has also been described by Nendwich et al. (2004), see also Valenti \& Fischer (2005).

\section{Conclusions}

It will certainly take additional time before model atmospheres for late-type stars are regularly constructed with physically more adequate assumptions than the standard ones - of spherical symmetry, mixing-length convection and LTE - adopted here. Until then, standard models will be the common choice in, e.g., abundance analyses. Here we have presented such a grid of standard models to the state of the art. It includes model atmospheres for stars of spectral types from $\mathrm{F}$ to $\mathrm{M}$, as well as carbon stars and Pop II stars of different metallicities. The assumptions, physical data used and numerical methods have been described, and

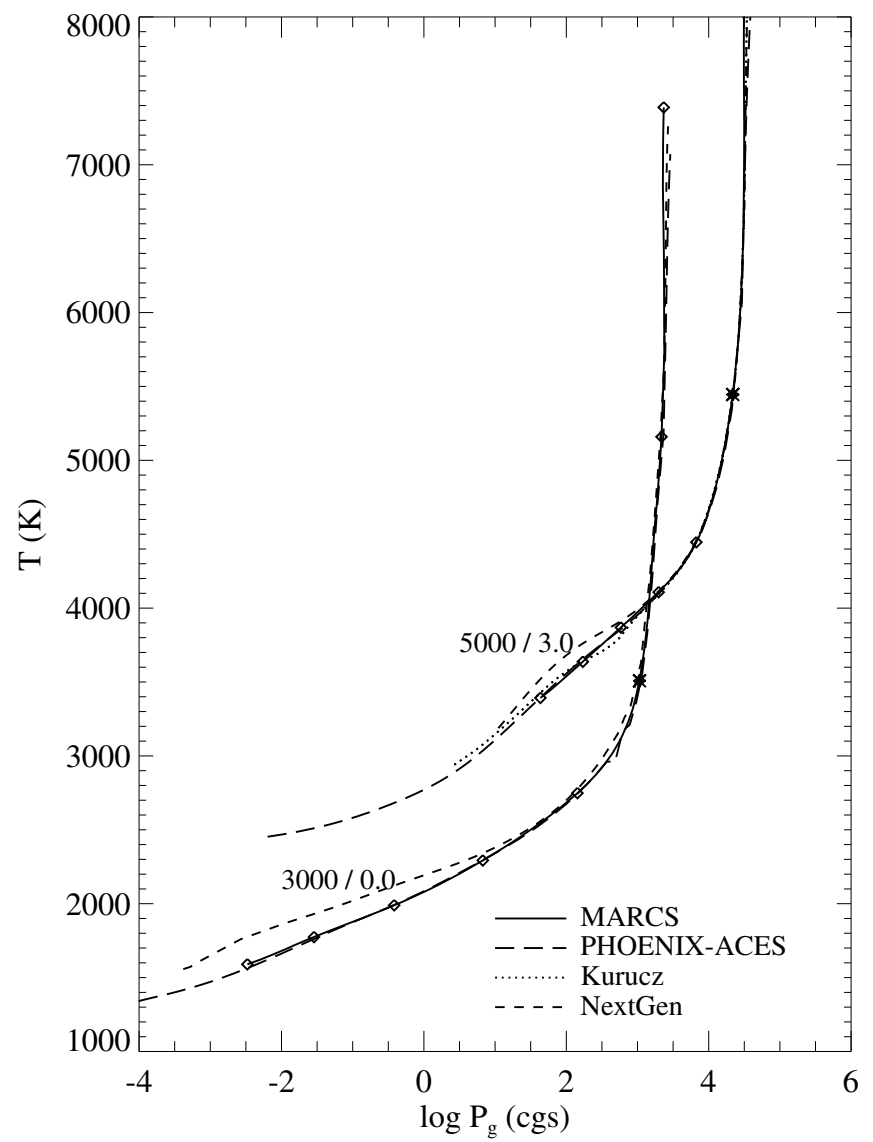

Fig. 19. Model atmospheres with solar abundances, from the MARCS, NextGen, and PHOENIX-ACES-2008 grids and (for $T_{\text {eff }}=5000 \mathrm{~K}$ ) the ODFNEW Castelli \& Kurucz grid. The $\log g$ parameter is $3.0(5000 \mathrm{~K})$ and $0.0(3000 \mathrm{~K})$, respectively. The MARCS and NextGen models are spherically symmetric with $M=5 M_{\odot}$ while the Kurucz model is planeparallel. Along the MARCS curve every decade in $\tau_{\text {Ross }}$ is marked by $\mathrm{a} \diamond$ sign, and $\tau_{\text {Ross }}=0.0$ by a $\times$. The general impression of a better agreement between MARCS and Castelli \& Kurucz models than between MARCS and NextGen models is also shown if the temperature is plotted relative to optical depth.

a number of general properties of the models have been illustrated. In particular, we have discussed the effects of blanketing and sphericity in some detail and tested several of the existing approximate analytical estimates of these effects and found them to work quite well. Also, already existing grids, such as the grid of plane-parallel models by Castelli \& Kurucz and the new PHOENIX grid, have been compared with and the agreement has been found to be excellent in the region of overlapping parameters. This is particularly gratifying in view of the fact that the different approaches, as regards numerical methods and codes and to a considerable degree also the selection of data, are independent. We may then basically view the calculation of standard 1D LTE model atmospheres as routine, but important improvements in line absorption data are still needed and certainly not easily achieved. This is also true for data for dust formation and dust opacities.

Further presentations and discussions of particular properties of these models will appear in subsequent papers in this series. Hopefully, these will also serve as starting points for further studies of the adequacy and shortcomings of the standard models, and as a stimulus for endeavours to set higher standards in the art of modelling stellar atmospheres in the future. 


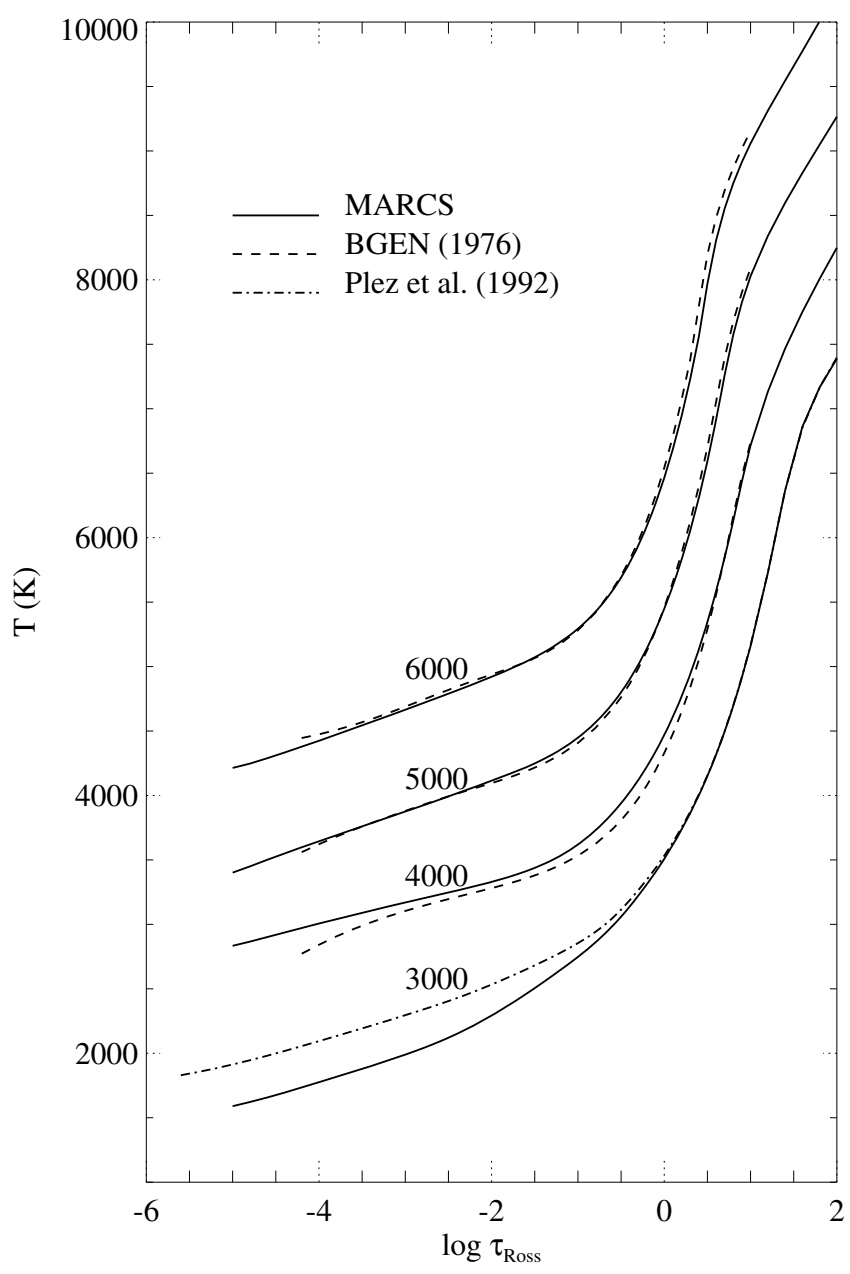

Fig. 20. Model atmospheres with solar abundances, from the present MARCS grid compared with corresponding MARCS models from Bell et al. (1976, BGEN, dashed) and with one model from Plez (1992). The $\log g$ parameter is 3.0 and the geometry is plane-parallel for the $6000 \mathrm{~K}, 5000 \mathrm{~K}$ and $4000 \mathrm{~K}$ models; the $3000 \mathrm{~K}$ models are spherically symmetric with $M=5 M_{\odot}$ and $\log g=0.0$.

Acknowledgements. We thank the Swedish Science Research Council and the Centre National de la Recherche Scientifique for long-standing support during the years. B.P. thanks Sveneric Johansson for his hospitality for several years at the Department for Physics at Lund. Previous collaborators in the development of the MARCS code are also thanked: Roger A Bell, Martin Asplund, John Brett, Leen Decin, Ulf Ekberg, Göran Hammarbäck, Dan Kiselman, Michelle Mizuno-Wiedner, Olle Morell, Nils Olander, Mikael Saxner and Nicole van der Bliek, as well as contributors and critics of important atomic and molecular data: Jan Almlöf, Bernhard Aringer, Paul Barklem, Manuel Bautista, Aleksandra Borysow, Alan Irwin, Per Jensen, Sveneric Johansson, Alain Jorissen, Bob Kurucz, David Lambert, Mats Larsson, Bo Lindgren, Nik Piskunov, Francois and Monique Querci, Jaques Sauval, Per Siegbahn and Georg Ole Sørensen. Peter Hauschildt is thanked for providing PHOENIX models for comparison and Thomas Masseron for providing his interpolation routine for MARCS models. Martin Asplund, Ulrike Heiter, Bob Kurucz, Jeff Linsky and Amy Mednick are thanked for valuable comments on the manuscript.

\section{References}

Alvarez, R., \& Plez, B. 1998, A\&A, 330, 1109

Alvarez, R., Lancon, A., Plez, B., et al. 2000, A\&A, 353, 322

Anderson, L. S. 1989, ApJ, 339, 558

Anstee, S. D., \& O'Mara, B. J. 1995, MNRAS, 276, 859

Aoki, W., Beers, T. C., Christlieb, N., et al. 2007, ApJ, 665, 492
Asplund, M., Gustafsson, B., Kiselman, D., \& Eriksson, K. 1997, A\&A, 323, 286

Asplund, M., Nordlund, Å., Trampedach, R., \& Stein, R. F. 1999, A\&A, 346, L17

Asplund, M., Nordlund, A., Trampedach, R., Allende Prieto, C., \& Stein, R. F. 2000a, A\&A, 359, 729

Asplund, M., Gustafsson, B., Lambert, D. L., \& Rao, N. K. 2000b, A\&A, 353, 287

Auer, L. H., \& Mihalas, D. 1970, MNRAS, 149, 65

Barber, R. J., Tennyson, J., Harris, G. J., \& Tolchenov, R. N. 2006, MNRAS, 368,1087

Barklem, P., \& Aspelund-Johansson, J. 2005, A\&A, 435, 373

Barklem, P., Piskunov, N., \& O’Mara, B. J. 2000a, A\&AS, 142, 467

Barklem, P., Piskunov, N., \& O’Mara, B. J. 2000b, A\&A, 363, 1091

Barklem, P., \& Piskunov, N. 2003, IAU Symp., 210, E28

Bautista, M. A. 1997, A\&AS, 122, 167

Bell, K. L., \& Berrington, K. A. 1987, J. Phys. B, 20, 801

Bell, K. L., Hibbert, K. A., \& Berrington, K. A. 1988, J. Phys. B, 21, 2319

Bell, R. A. 1971, MNRAS, 154, 343

Bell, R. A., \& Gustafsson, B. 1989, MNRAS, 236, 653

Bell, R. A., Eriksson, K., Gustafsson, B., \& Nordlund, A. 1976, A\&AS, 23, 37

Bensby, T., Feltzing, S., Lundström, I., \& Ilyiin, I. 2005, A\&A, 433, 185

Bessell, M. S., Castelli, F., \& Plez, B. 1998, A\&A, 333, 231; A\&A, 337, 321

Blackwell, D. E., Booth, A. J., Petford, A. D., et al. 1989, MNRAS, 236, 235

Böhm-Vitense, E. 1972, A\&A, 17, 335

Boothroyd, A. I., \& Sackmann, I.-J. 1999, ApJ, 510, 232

Borysow, A., Jørgensen, U. G., \& Fu, Y. 2001, JQRST, 68, 235

Bowen, G. 1988, ApJ, 329, 299

Brault, J., \& Neckel, H. 1987, Spectral Atlas of Solar Disk-averaged and Disk-center Intensity from 3290 to $12510 \AA$, unpublished (electronic copy by anonymous ftp from ftp.hs.uni-hamburg.de; Directory /pub/outgoing/fts-atlas)

Brett, J. M., \& Plez, B. 1993, PASAu, 10, 250

Bues, I., \& Wehrse, R. 1976, A\&A, 51, 461

Carbon, D. F. 1979, ARA\&A, 17, 513

Carbon, D. F., \& Gingerich, O. 1969, Proc. 3rd Harvard-Smithsonian Conf. on Stellar Atmospheres, ed. O. Gingerich, Cambridge, Massachusettes Inst. of Technology, 377

Castelli, F., \& Kurucz, R. L. 2003, IAU Symp., 210, A20 [arXiv: astro-ph/0405087]

Castelli, F., Gratton, R. G., \& Kurucz, R. L. 1997, A\&A, 318, 841; A\&A, 324, 432

Cayrel, R., Depagne, E., Spite, M., et al. 2004, A\&A, 416, 1117

Charbonnel, C. 1994, A\&A, 282, 811

Christlieb, N., Gustafsson, B., Korn, A. J., et al. 2004, ApJ, 603, 708

Dalgarno, A., \& Williams, D. A. 1962, ApJ, 136, 690

Däppen, W., Anderson, L., \& Mihalas, D. 1987, ApJ, 319, 195

Decin, L., Waelkens, C., Eriksson, K., et al. 2000, A\&A, 364, 137

Decin, L., Vanderbussche, B., Waelkens, C., et al. 2003, A\&A, 400, 709

Doyle, R. O. 1968, ApJ, 153, 987

Edvardsson, B., Andersen., J., Gustafsson, B., et al. 1993, A\&A, 275, 101

Edvardsson, B., Eriksson, K., Gustafsson, B., et al. 2008, A\&A, to be submitted

Ekberg, U., Eriksson, K., \& Gustafsson, B. 1986, A\&A, 167, 304

Feautrier, P. 1964, C.R. Acad. Sci. 258, 3189

Fleischer, A. J., Gauger, A., \& Sedlmayr, E. 1992, A\&A, 266, 321

Fluks, M. A., Plez, B., The, P. S., et al. 1994, A\&AS, 105, 311

Frebel, A., Aoki, W., Christlieb, N., et al. 2005, Nature, 434, 871

Freytag, B. 2001, in 11th Cambridge Workshop on Cool Stars, Stellar Systems and the Sun (ASP: San Francisco), 785

Fuhrmann, K. 1998, A\&A, 338, 161

Gibson, G. E., \& Heitler, W. 1928, Z. Phys., 49, 465

Gillis, J. R., Goldman, A., Stark, G., et al. 2001, JQSRT, 68, 225

Gingerich, O. 1964, SAO Spec. Rep., 167

Goldman, A., Schoenfeld, W. G., Goorvitch, D., et al. 1998, JQSRT, 59, 453

Goorvitch, D. 1994, ApJS, 95, 535

Grevesse, N., \& Sauval, A. J. 1998, Space Science Rev., 85, 161

Grevesse, N., Asplund, M., \& Sauval, A. J. 2007, Space Sci. Rev., 130, 105

Gustafsson, B., \& Bell, R. A. 1979, A\&A, 74, 313

Gustafsson, B., \& Jørgensen, U. G. 1994, A\&AR, 6, 19

Gustafsson, B., \& Nissen, P. E. 1972, A\&A, 19, 261

Gustafsson, B., \& Olander, N. 1979, Phys. Scr., 20, 570

Gustafsson, B., \& Plez, B. 1992, Proc. International Coll., ed. C. de Jager, \& H. Nieuwenhuijzen (Amsterdam: North Holland), 86

Gustafsson, B., Bell, R. A., Eriksson, K., \& Nordlund, ̊.. 1975, A\&A, 42, 407

Gustafsson, M., \& Frommhold, L. 2001, ApJ, 546, 1168

Gustafsson, M., \& Frommhold, L. 2003, A\&A, 400, 1161

Harris, G. J., Polyansky, O., \& Tennyson, J. 2002, ApJ, 578, 657 
Hartman, H., Derkatch, A., Donnelly, M. P., et al. 2003, A\&A, 397, 1143

Hauschildt, P. H., Allard, F., Ferguson, J., et al. 1999, ApJ, 525, 871

Hauschildt, P. H., Allard, F., Barman, T., et al. 2002, in Galactic Structure, Stars and the Interstellar Medium, ed. C. Woodward, M. Bicay, \& J. Shull (ASP San Francisco), 427

Heiter, U., \& Eriksson, K. 2006, A\&A, 452, 1039

Henyey, L., Vardya, M. S., \& Bodenheimer, P. 1965, ApJ, 142, 841

Hill, V., Barbuy, B., Spite, M., et al. 2000, A\&A, 353, 557

Holweger, H., \& Müller, E. A. 1974, Sol. Phys., 39, 19

Höfner, S. 1999, A\&A, 346, L9

Höfner, S., \& Dorfi, E. A. 1997, A\&A, 319, 648

Höfner, S., Gautschy-Loidl, R., Aringer, B., \& Jørgensen, U. G. 2003, A\&A, 399, 589

Irwin, A. W. 1981, ApJS, 45, 621

Irwin, A. W. 1988, A\&AS, 74, 145

John, T. L. 1975a, MNRAS, 170, 5

John, T. L. 1975b, MNRAS, 172, 305

John, T. L. 1994, MNRAS, 269, 871

John, T. L., \& Williams, R. J. 1975, MNRAS, 171, 7

Johnson, H. R. 1973, ApJ, 180, 81

Johnson, H. R., Bernat, A. P., \& Krupp, B. M. 1980, ApJS, 42, 501

Jørgensen, U. G. 1989, ApJ, 344, 901

Jørgensen, U. G. 1990, A\&A, 232, 420

Jørgensen, U. G. 1994a, A\&A, 284, 179

Jørgensen, U. G. 1994b, Proc. IAU Coll., 146, ed. U. G. Jørgensen (New-York, Berlin, Heidelberg: Springer-Verlag), 29

Jørgensen, U. G. 1997, IAU Symp., 178, 441

Jørgensen, U. G. 2005, ASPC, 336, 269

Jørgensen, U. G., \& Larsson, M. 1990, A\&A, 238, 424

Jørgensen, U. G., \& Johnson, H. R. 1992, A\&A, 265, 168

Jørgensen, U. G., Almlöf, J., Gustafsson, B., et al. 1985, J. Chem. Phys., 83, 3034

Jørgensen, U. G., Almlöf, J., \& Siegbahn, P. E. M. 1989, ApJ, 343, 554

Jørgensen, U. G., Johnson, H. R., \& Nordlund, ^.. 1992, A\&A, 261, 263

Jørgensen, U. G., Larsson, M., Iwamae, A., \& Yu, B. 1996, A\&A, 315, 204

Jørgensen, U. G., Hammer, D., Borysow, A., et al. 2000, A\&A, 361, 283

Jørgensen, U. G., Jensen, P., Sørensen, G. O., \& Aringer, B. 2001, A\&A, 372, 249

Karzas, W. J., \& Latter, R. 1961, ApJS, 6, 167

Krupp, B. M., Collins, J. G., \& Johnson, H. R. 1978, ApJ, 219, 963

Kurucz, R. L. 1979, ApJS, 40, 1

Kurucz, R. L. 1995a, Kurucz CD-ROM, 15, Cambridge, Mass.: Smithsonian Astrophysical Observatory

Kurucz, R. L. 1995b, Laboratory and Astronomical High Resolution Spectra, ed. A. J. Sauval, R. Blomme, \& N. Grevesse, ASP Conf. Ser., 81, 583

Kurucz, R. L., \& Bell, B. 1995, Kurucz CD-ROM No. 23, Cambridge, Mass: Smithsonian, Astrophysical Observatory

Kurucz, R. L., van Dishoeck, E. F., \& Tarafdar, S. P. 1987, ApJ, 322, 992

Lambert, D. L., Gustafsson, B., Eriksson, K., \& Hinkle, K. H. 1986, ApJS, 62, 373

Langhoff, S. R., \& Bauschlicher, C. W., Jr. 1993, Chem. Phys. Lett., 211, 305

Lawler, J. E., Hartog, E. A. D., Labby, Z. E., et al. 2007, ApJS, 169, 120

Lederer, M. T., Lebzelter, T., Aringer, B., et al. 2006, Mem. S.A.It, 77, 1008

Loidl, R., Lancon, A., \& Jørgensen, U. G. 2001, A\&A, 371, 1065
Masseron, T. 2006, Ph.D. Thesis, Obs. de Paris

Massey, P., Plez, B., Levesque, E. M., et al. 2007, [arXiv: 0708. 2847]

Mihalas, D. 1964, ApJS, 9, 321

Mihalas, D. 1978, Stellar Atmospheres, 2nd edn. (W. H. Freeman and Co., San Francisco)

Nave, G., Johansson, S., Learner, R. C. M., et al. 1994, ApJS, 94, 221

Nendwich, J., Heiter, U., Kupka, F., et al. 2004, Comm. Astroseismol., 144, 43

Nissen, P. E., Chen, Y. Q., Asplund, M., et al. 2004, A\&A, 415, 993

Nordlund, A. 1974, A\&A, 32, 407

Nordlund, A. 1982, A\&A, 107, 1

Nordlund, Å. 1984, in Methods in Radiative Transfer, ed. W. Kalkofen (Cambridge University Press), 211

Nordlund, A., \& Dravins, D. 1990, A\&A, 228, 155

Norris, J. E., Christlieb, N., Korn, A., et al. 2007, ApJ, 670, 774

Olander, N. 1981, Ph.D. Thesis, Uppsala University

Peach, G. 1970, MmRAS, 73, 1

Peytremann, E. 1974, A\&A, 33, 203

Piskunov, N. E., Kupka, F., Ryabchikova, T. A., Weiss, W. W., \& Jeffery, C. S. 1995, A\&AS, 112, 525 (VALD-1)

Plez, B. 1990, MmSAI, 61, 765

Plez, B. 1992, A\&AS, 94, 527

Plez, B. 1998, A\&A, 337, 495

Plez, B., Brett, J. M., \& Nordlund, A. 1992, A\&A, 256, 551

Plez, B., Van Eck, S., Jorissen, A., et al. 2003, IAU Symp., 210, A2

Plez, B., Masseron, T., Van Eck, et al. 2008, Identification of near-UV predissociation lines of $\mathrm{CH}$ in carbon-enhanced Fe-poor stars, in Cool Star, Stellar Systems, and the Sun, ed. G. van Belle, ASP Conf. Ser., in press

Querci, F., Querci, M., \& Kunde, V. 1971, A\&A, 15, 256

Querci, F., Querci, M., \& Tsuji, T. 1974, A\&A, 31, 265

Ramsbottom, C. A., Bell, K. L., \& Berrington, K. A. 1992, J. Phys. B, 25, 1443

Reddy, B. E., Tomkin, J., Lambert, D. L., \& Allende Prieto, C. 2003, MNRAS, 340,304

Russell, H. N. 1934, ApJ, 79, 317

Rybicki, G. B. 1971, JQSRT, 11, 589

Ryde, N., \& Lambert, D. L. 2004, A\&A, 415, 559

Sauval, A. J., \& Tatum, J. B. 1984, ApJS, 56, 193

Saxner, M., \& Gustafsson, B. 1984, A\&A, 140, 334

Schmid-Burgk, J., \& Scholz, M. 1975, A\&A, 41, 41

Schmid-Burgk, J., Scholz, M., \& Wehrse, R. 1981, MNRAS, 194, 383

Seaton, M. J., Yan, Y., Mihalas, D., \& Pradhan, A. K. 1994, MNRAS, 266, 805

Skory, S., Weck, P. F., Stancil, P. C., et al. 2003, ApJS, 148, 599

Sneden, C., Johnson, H. R., \& Krupp, B. M. 1976, ApJ, 204, 281

Stempels, H. C., Piskunov, N., \& Barklem, P. S. 2001, Proc. 11th Cambridge

Workshop on Cool Stars, Stellar Systems and the Sun, ed. R. J. Garcia Lopez,

R. Rebolo, \& M. R. Zapeterio Osorio, ASP Conf. Ser., 223, 878 (VALD-2)

Stein, R. F., \& Nordlund, A. 1998, ApJ, 499, 914

Unsöld, A. 1955, Physik der Sternatmosfären, 2nd edn. (Berlin, Göttingen, Heidelberg: Springer-Verlag)

Valenti, J. A., \& Fischer, D. A. 2005, ApJS, 159, 141

Valenti, J. A., \& Piskunov, N. 1996, A\&AS, 118, 595

van der Bliek, N. S., Gustafsson, B., \& Eriksson, K. 1996, A\&A, 309, 849

Venn, K. A., Irwin, M., Shetrone, M. D., et al. 2004, AJ, 128, 1177

Wishart, A. W. 1979, MNRAS, 187, 59

Wood, P. R. 1979, ApJ, 227, 220 